\title{
Black hole evolution by spectral methods
}

\author{
Lawrence E. Kidder, Mark A. Scheel, and Saul A. Teukolsky \\ Center for Radiophysics and Space Research, Cornell University, Ithaca, New York 14853 \\ Eric D. Carlson and Gregory B. Cook \\ Department of Physics, Wake Forest University, Winston-Salem, North Carolina 27109
}

(Received 15 May 2000; published 26 September 2000)

\begin{abstract}
Current methods of evolving a spacetime containing one or more black holes are plagued by instabilities that prohibit long-term evolution. Some of these instabilities may be due to the numerical method used, traditionally finite differencing. In this paper, we explore the use of a pseudospectral collocation (PSC) method for the evolution of a spherically symmetric black hole spacetime in one dimension using a hyperbolic formulation of Einstein's equations. We demonstrate that our PSC method is able to evolve a spherically symmetric black hole spacetime forever without enforcing constraints, even if we add dynamics via a Klein-Gordon scalar field. We find that, in contrast with finite-differencing methods, black hole excision is a trivial operation using PSC applied to a hyperbolic formulation of Einstein's equations. We discuss the extension of this method to three spatial dimensions.
\end{abstract}

PACS number(s): 04.25.Dm, 02.70.Hm

\section{INTRODUCTION AND SUMMARY}

A major thrust of research in classical general relativity in the past decade has been to devise algorithms to solve Einstein's equations numerically. Despite advances in our analytic understanding of general relativity, we still do not know what all the features of the theory really are. Numerical solutions will continue to provide fresh insights into the theory as they have in the past, for example, the critical behavior in black hole formation [1] and the formation of toroidal black holes [2].

New urgency has been injected into numerical relativity by the imminent deployment of the Laser Interferometric Gravitational Wave Observatory (LIGO). The prime target for LIGO is coalescence of binary neutron star and black hole systems. The waveform is reasonably well predicted by the post-Newtonian approximation when the binary components are at large separation. However, extracting the most important physics requires us to be able to deal with fully non-linear general relativity as the system spirals together and coalesces. Moreover, a number of people believe that there is a significant event rate for the coalescence of massive black hole systems $\left(\sim 20 M_{\odot}\right)$ [3]. In this case, LIGO is most sensitive to waves emitted from the strong field regime. Indeed, without some theoretical guidance as to what to expect from this regime, it is possible we may miss these events entirely [4].

However, the goal of developing a general algorithm that can solve Einstein's equations for two black holes has remained elusive. All attempts to date have been plagued by instabilities. These instabilities are caused by an interplay of three factors: (1) Einstein's equations are an overdetermined system, with the evolution equations subject to constraints. So if, for example, you choose to solve only the evolution equations, then there can be unstable solutions that are in fact solutions of the evolution equations, but do not satisfy the constraints. Small numerical errors may cause these solutions to appear and swamp the true solution ("constraint- violating modes"). (2) The coordinate freedom inherent in the theory means that it is very easy to impose coordinate conditions that lead to numerical instabilities ("gauge modes"). (3) Experience has shown that the kind of boundary conditions we choose and how we implement them can affect the stability of an algorithm enormously.

Similar instabilities have hampered efforts to solve the related problem of binary neutron stars; only very recently $[5,6]$ has there been some success in finding stable algorithms. However, black hole evolutions face an additional obstacle that is absent in the case of neutron stars: for neutron stars the gravitational field is everywhere regular, but for black holes one must somehow deal with the physical singularity that lurks inside each hole.

There are two main approaches for handling these singularities. The first is to use gauge conditions (e.g., maximal slicing) that avoid the singularities altogether. Such conditions, however, lead to large gradients in the gravitational field variables near the horizon. These grow exponentially in time and ultimately cannot be resolved by the numerical evolution, causing the code to crash. The alternative approach is to excise the region containing the singularity from the computational domain and evolve only the exterior region. If the excision boundary is placed inside the horizon of the black hole, causality assures us that we do not need to impose a physical boundary condition there.

However, black hole excision is only known to be mathematically well posed if the evolution equations are hyperbolic with characteristic speeds less than or equal to $c$. In this case, the structure of the equations guarantees that even unphysical modes present in the solution (gauge modes, constraint-violating modes) behave causally and cannot propagate out of the horizon. For many representations of general relativity such as the usual Arnowitt-Deser-Misner (ADM) [7] formulation, the evolution equations are of no mathematical type for which well-posedness has been proved, so the suitability of these formulations for black hole excision must be determined empirically on a case-by-case 
basis. It is in part for this reason that much attention has been recently focused on hyperbolic representations of Einstein's equations [8-19].

It is still unclear whether hyperbolic formulations are computationally advantageous. However, it has been shown that the formulation of the evolution equations can affect stability. For example, some instabilities can be eliminated by changing from one formulation of Einstein's equations to another [20] or by modifying the evolution equations to change the spectrum of unphysical modes [21].

Nevertheless, it is likely that many instabilities encountered in practice are due to the numerical implementation of the evolution equations and of the boundary conditions. Even for well-understood systems, it is far from guaranteed that any given numerical approximation to the continuum equations will be stable. The well-known Courant instability is a trivial example. Hence it is prudent to explore alternative numerical methods that may offer a shorter path to the goal of long-term stability.

Traditionally, black hole spacetimes have been evolved using finite-difference (FD) methods. Current FD codes for evolving black hole spacetimes with excised horizons are mostly based on a numerical technique known as causal differencing [22-27], which allows one to update the fundamental variables in time while avoiding numerical problems associated with superluminal grid speeds. This technique has been used successfully to propagate an excised hole across a grid, even when grid points fall into or emerge from the horizon [25].

However, causal differencing is complicated because it requires interpolation, and it has to deal with points "missing" in some irregular fashion near the excision boundary. The FD operator that performs the interpolation depends on the shape of the excision boundary. Furthermore, even for a given excision boundary and a given target point, the operator is not unique. Hence one must construct a large number of interpolation operators, each of which involves some arbitrary choice, in order to perform interpolations on the entire grid. It is only by trial and error that one finds operators that result in a stable evolution scheme. For simulations of a single spherical black hole on a Cartesian three-dimensional grid, we have found a case in which changing a single interpolation operator that is used only for a single target point on the grid makes the difference between a stable and an unstable code.

Another limitation of FD methods is the difficulty of imposing boundary conditions at the outer boundary of the calculation. There are two aspects to this problem: First, one must formulate a procedure for handling the boundary, such as imposing an analytic condition (e.g., a Sommerfeld condition) on the fundamental variables, matching to a wave perturbation described by the Zerilli equation [28] or matching to a characteristic evolution code that propagates the solution out to null infinity [29-31]. Second, one must construct a FD approximation of either the analytic boundary condition or the matching condition. It can be difficult to find such an approximation that yields a stable evolution.

In this paper we explore an alternative computational strategy: a pseudospectral collocation (PSC) scheme. In our
PSC evolution scheme, the solutions to a set of hyperbolic differential equations are approximated as series expansions in a set of orthogonal basis functions (e.g., Chebyshev polynomials) in space, and these coefficients are integrated forward in time using the method of lines. PSC has three important advantages over FD: (1) No ad hoc interpolation operators are required to determine field values at an arbitrary point because the solution provided by PSC is an analytic function given everywhere on the computational domain. (2) Boundary conditions are imposed directly on the basis functions, with no approximations, in a straightforward manner. (3) For smooth solutions, PSC will converge to the actual solution exponentially as the number of basis functions is increased. A FD solution, on the other hand, never converges faster than algebraically with the number of grid points. Thus, for a given accuracy, PSC requires far less CPU time and memory than FD methods.

One of the drawbacks of using PSC for hyperbolic problems is a more restrictive Courant stability limit than for FD. For the wave equation, a PSC method using $N$ Chebyshev polynomials requires $\Delta t \lesssim O\left(N^{-2}\right)$, whereas typical FD methods with $N$ grid points require $\Delta t \leqq O\left(N^{-1}\right)$. However, this is not as severe a restriction as it may seem at first glance because for a given accuracy $N$ is much smaller for PSC than for FD. Moreover, if the time step becomes too small for practical evolutions, one can implement implicit or semiimplicit time-stepping schemes, which have no Courant stability limit.

Another difficulty commonly encountered in PSC methods is the Gibbs phenomenon: because the spectral coefficients of a discontinuous function do not vanish at high frequencies, spectral methods typically produce nonconvergent oscillitory solutions when applied to problems involving discontinuities. Fortunately, for black holes we should have only smooth solutions, so the Gibbs phenomenon should not play a role. Any discontinuities that may arise from poor gauge choices [32] represent a breakdown of the coordinate system and should terminate the evolution regardless of whether one uses PSC. Furthermore, we do not anticipate using discontinuous boundary conditions (for which a Gibbs phenomenon can also occur), even in three dimensions.

There are also instabilities associated with PSC methods that one does not encounter in FD. These are typically associated with aliasing errors (see Sec. III G) and can often be eliminated by periodically smoothing the solution [33]. These instabilities have not caused much difficulty for the spherically symmetric evolutions reported here, but our preliminary efforts in three dimensions show that more extensive smoothing may be necessary for solutions on multiple domains.

PSC has been applied successfully to solve problems in many fields, including fluid dynamics, meteorology, seismology, and relativistic astrophysics (cf. [34-37]). For example, PSC has been applied successfully to model stellar core collapse [38] and construct equilibrium sequences of irrotational binary neutron stars [39]. For black hole spacetimes PSC has been applied successfully to solve initial data for the stan- 
dard field equations [40] and the conformal field equations [41], to find apparent horizons [42], to solve the shift vector equation for a Kerr black hole [43], and to evolve Einstein's equations in null quasi-spherical coordinates [44].

Here we evolve a spherically symmetric black hole spacetime in one spatial dimension by applying PSC methods to a hyperbolic formulation of Einstein's equations, the "Einstein-Christoffel" (EC) system [19]. A hyperbolic formulation provides a well-defined prescription for imposing boundary conditions. At a boundary, the fundamental fields can be decomposed into characteristic fields, which in the case of the EC system propagate either along the light cone or normal to the spatial foliation. Boundary conditions are imposed on the incoming (with respect to the computational domain) characteristic fields, but not on the outgoing fields. After imposing the boundary condition, the fundamental fields are reconstituted from the characteristic decomposition. If the excision boundary is placed inside the event horizon of a black hole, then (for appropriate coordinate systems) all characteristic fields are outgoing at this boundary, so no boundary conditions are needed there. Thus, black hole excision is a trivial operation. At the outer boundary of the domain, boundary conditions corresponding to no incoming radiation can be imposed on the incoming characteristic fields.

We find that our PSC method is able to evolve a spherically symmetric black hole spacetime forever. Furthermore, we find that the solution converges exponentially to the exact solution as the number of basis functions is increased. We discuss the time-stepping algorithms, outer boundary conditions, and gauge conditions required for stable evolution and how these depend on the particular slicing of the Schwarzschild geometry we wish to reproduce. We also show that our PSC method can handle dynamics by evolving a black hole spacetime containing a scalar field. Finally, we outline a strategy for applying this method to two black holes in three spatial dimensions using multiple domains, and we present tests of this domain decomposition idea in spherical symmetry.

In Sec. II we list the basic equations we use to evolve a spherically symmetric spacetime, boundary conditions, gauge conditions, initial data, and diagnostics. In Sec. III we introduce PSC and describe our numerical methods. In Sec. IV we present numerical evolutions of single black hole spacetimes in spherical symmetry. Finally, in Sec. V we dis- cuss our results and the generalization of our methods to multiple dimensions and to binary black hole spacetimes.

\section{BASIC EQUATIONS}

\section{A. Einstein-Christoffel system}

We adopt the "Einstein-Christoffel" hyperbolic representation of Einstein's equations [19]. In this formulation the evolution equations are written in first-order symmetric hyperbolic form, and all characteristic curves are directed either along the light cone or normal to the spatial foliation. The EC system takes as fundamental quantities the familiar threemetric and extrinsic curvature plus only 18 additional "connection" variables (in three spatial dimensions). Unlike some hyperbolic representations of general relativity, the EC system requires no derivatives of the stress-energy tensor.

We write the metric in the usual $3+1$ form

$$
d s^{2}=-N^{2} d t^{2}+g_{i j}\left(d x^{i}+\beta^{i} d t\right)\left(d x^{j}+\beta^{j} d t\right),
$$

where $g_{i j}$ is the three-metric, $\beta^{i}$ is the shift vector, and $N$ is the lapse function. In the EC formulation it is not the lapse function $N$ that is freely specifiable; instead, one arbitrarily prescribes the densitized lapse function $\alpha$, defined by

$$
\alpha \equiv \frac{N}{\sqrt{g}},
$$

where $g$ is the determinant of the three-metric. The use of a densitized lapse does not fix the temporal gauge freedom in any way: one can in principle obtain any lapse function $N$ by an appropriate choice of $\alpha$.

To write down the EC evolution equations, first define the new variables

$$
f_{k i j} \equiv \Gamma_{(i j) k}+g_{k i} g^{l m} \Gamma_{[l j] m}+g_{k j} g^{l m} \Gamma_{[l i] m},
$$

where $\Gamma^{k}{ }_{i j}$ is the affine connection associated with $g_{i j}$, and parentheses and brackets denote symmetrization and antisymmetrization, respectively. The quantities $f_{k i j}$ will be taken as fundamental variables along with $g_{i j}$ and the extrinsic curvature $K_{i j}$.

The EC evolution equations can be written in the form

$$
\begin{aligned}
\widehat{\partial_{0} g_{i j}=} & -2 N K_{i j}, \\
\widehat{\partial_{0} K_{i j}+N g^{k l} \partial_{l} f_{k i j}=} & N\left\{g^{k l}\left(K_{k l} K_{i j}-2 K_{k i} K_{l j}\right)+g^{k l} g^{m n}\left(4 f_{k m i} f_{[l n] j}+4 f_{k m[n} f_{l] i j}-f_{i k m} f_{j l n}\right.\right. \\
& \left.+8 f_{(i j) k} f_{[l n] m}+4 f_{k m(i} f_{j) l n}-8 f_{k l i} f_{m n j}+20 f_{k l(i} f_{j) m n}-13 f_{i k l} f_{j m n}\right) \\
& -\partial_{i} \partial_{j} \ln \alpha-\left(\partial_{i} \ln \alpha\right)\left(\partial_{j} \ln \alpha\right)+2 g_{i j} g^{k l} g^{m n}\left(f_{k m n} \partial_{l} \ln \alpha-f_{k m l} \partial_{n} \ln \alpha\right) \\
& \left.+g^{k l}\left[\left(2 f_{(i j) k}-f_{k i j}\right) \partial_{l} \ln \alpha+4 f_{k l(i} \partial_{j)} \ln \alpha-3\left(f_{i k l} \partial_{j} \ln \alpha+f_{j k l} \partial_{i} \ln \alpha\right)\right]-8 \pi S_{i j}+4 \pi g_{i j} T\right\},
\end{aligned}
$$




$$
\begin{aligned}
\widehat{\partial_{0}} f_{k i j}+N \partial_{k} K_{i j}= & N\left\{g^{m n}\left[4 K_{k(i} f_{j) m n}-4 f_{m n(i} K_{j) k}+K_{i j}\left(2 f_{m n k}-3 f_{k m n}\right)\right]\right. \\
& +2 g^{m n} g^{p q}\left[K_{m p}\left(g_{k(i} f_{j) q n}-2 f_{q n(i} g_{j) k}\right)+g_{k(i} K_{j) m}\left(8 f_{n p q}-6 f_{p q n}\right)\right. \\
& \left.+K_{m n}\left(4 f_{p q(i(i) k} g_{j) k}-5 g_{k(i} f_{j) p q}\right)\right]-K_{i j} \partial_{k} \ln \alpha \\
& \left.+2 g^{m n}\left(K_{m(i} g_{j) k} \partial_{n} \ln \alpha-K_{m n} g_{k(i} \partial_{j)} \ln \alpha\right)+16 \pi g_{k(i} J_{j)}\right\} .
\end{aligned}
$$

Here the symbol $\widehat{\partial_{0}}$ is the time derivative operator normal to the spatial foliation, defined by

$$
\widehat{\partial_{0}} \equiv \partial_{t}-£_{\beta},
$$

where $£$ denotes a Lie derivative. The matter terms are

$$
\begin{gathered}
\rho \equiv n^{\mu} n^{\nu} T_{\mu \nu}, \\
T \equiv{ }^{(4)} g^{\mu \nu} T_{\mu \nu}, \\
J_{i} \equiv-n^{\mu} \gamma_{i}{ }^{\nu} T_{\mu \nu}, \\
S_{i j} \equiv \gamma_{i}{ }^{\nu} \gamma_{j}^{\mu} T_{\mu \nu},
\end{gathered}
$$

where $T_{\mu \nu}$ is the stress-energy tensor, ${ }^{(4)} g_{\mu \nu}$ is the fourmetric, $n^{\mu}$ is the unit normal to the spatial foliation, and $\gamma_{i}{ }^{\nu}$ is the spatial projection operator $n^{\nu} n_{i}+{ }^{(4)} g_{i}{ }^{\nu}$.

Note that for each $\{i, j\}$ pair, the evolution equations (2.4) can be written in the form (dropping the $i$ and $j$ indices on $g_{i j}, K_{i j}$, and $f_{k i j}$ )

$$
\begin{aligned}
\widehat{\partial_{0}} g & =-2 N K, \\
\widehat{\partial_{0}} K+N g^{k l} \partial_{l} f_{k} & =R, \\
\widehat{\partial_{0}} f_{k}+N \partial_{k} K & =S,
\end{aligned}
$$

where $R$ and $S$ are nonlinear terms that contain no derivatives of the fundamental variables (but may contain spatial derivatives of the arbitrary gauge function $\alpha$ ). Except for the righthand sides, Eqs. (2.7a) are just $\square g=0$ written in first order form. Thus one can think of the EC system (2.4) as a set of six (one for each $\{i, j\}$ pair) coupled quasilinear scalar wave equations with nonlinear source terms.

A solution of the evolution equations (2.4) is not a solution to Einstein's equations unless 22 constraints are also satisfied. These are the Hamiltonian constraint

$$
\begin{aligned}
\mathcal{C} \equiv & g^{i j} g^{k l}\left\{2\left(\partial_{k} f_{i j l}-\partial_{i} f_{j k l}\right)+K_{i k} K_{j l}-K_{i j} K_{k l}\right. \\
& +g^{m n}\left[f_{i k m}\left(5 f_{j l n}-6 f_{l j n}\right)+13 f_{i k l} f_{j m n}\right. \\
& \left.\left.+f_{i j k}\left(8 f_{m l n}-20 f_{l m n}\right)\right]\right\}+16 \pi \rho=0,
\end{aligned}
$$

the 3 momentum constraints

$$
\begin{aligned}
\mathcal{C}_{i} \equiv & g^{k l}\left\{g^{m n}\left[K_{i k}\left(3 f_{l m n}-2 f_{m n l}\right)-K_{k m} f_{i l n}\right]\right. \\
& \left.+\partial_{i} K_{k l}-\partial_{k} K_{i l\}}\right\}+8 \pi J_{i}=0,
\end{aligned}
$$

and the 18 constraints

$$
\mathcal{C}_{k i j} \equiv \partial_{k} g_{i j}-2 f_{k i j}+4 g^{l m}\left(f_{l m(i} g_{j) k}-g_{k(i} f_{j) l m}\right)=0,
$$

which relate $f_{k i j}$ to spatial derivatives of the three-metric. If the constraints are satisfied for the initial data, they are preserved by the evolution equations for all time. As for any formulation of Einstein's equations, however, numerical approximations may spoil this constraint-preserving property.

\section{B. Einstein-Klein-Gordon system}

This paper is primarily concerned with vacuum black holes, $T_{\mu \nu}=0$. However, to test our spherically symmetric code in a dynamical spacetime, in Sec. IV B we include a Klein-Gordon scalar field $\phi$ with stress-energy

$$
4 \pi T_{a b} \equiv\left(\partial_{a} \phi\right)\left(\partial_{b} \phi\right)-\frac{1}{2} g_{a b}\left(\partial_{c} \phi\right)\left(\partial^{c} \phi\right) .
$$

Defining the quantities

$$
\begin{aligned}
\Pi & \equiv-N^{-1} \widehat{\partial_{0}} \phi, \\
\Phi_{i} & \equiv \partial_{i} \phi,
\end{aligned}
$$

the matter terms (2.6) are given by

$$
\begin{aligned}
4 \pi \rho & =\frac{1}{2}\left(\Pi^{2}+\Phi^{i} \Phi_{i}\right), \\
4 \pi J_{i} & =\Pi \Phi_{i}, \\
4 \pi T & =\Pi^{2}-\Phi^{i} \Phi_{i}, \\
4 \pi S_{i j} & =\Phi_{i} \Phi_{j}+\frac{1}{2} g_{i j}\left(\Pi^{2}-\Phi^{i} \Phi_{i}\right) .
\end{aligned}
$$

The scalar field obeys $\square \phi=0$, which one can write in the $3+1$ first-order form

$$
\widehat{\partial_{0}} \phi=-N \Pi,
$$

$$
\begin{aligned}
\widehat{\partial_{0}} \Pi+N g^{i j} \partial_{i} \Phi_{j}= & N g^{i j}\left\{\Pi K_{i j}-\Phi_{i} \partial_{j} \ln \alpha\right. \\
& \left.+4 \Phi^{k} f_{[k i] j}\right\}, \\
\widehat{\partial_{0}} \Phi_{i}+N \partial_{i} \Pi= & -N \Pi\left\{\partial_{i} \ln \alpha\right. \\
& \left.+g^{j k}\left(3 f_{i j k}-2 f_{k i j}\right)\right\} .
\end{aligned}
$$


Equations (2.12) and (2.4) form a larger hyperbolic system of evolution equations. Note that if one evolves $\Pi$ and $\Phi_{i}$, it is not necessary to also evolve $\phi$ because $\phi$ appears neither in Eqs. (2.11) nor in the evolution equations for $\Pi$ and $\Phi_{i}$.

\section{Reduction to spherical symmetry}

The most general spherically symmetric metric can be written in the form

$$
d s^{2}=-N^{2} d t^{2}+g_{r r}\left(d r+\beta^{r} d t\right)^{2}+g_{T} r^{2}\left(d \theta^{2}+\sin ^{2} \theta d \phi^{2}\right),
$$

where the transverse metric component is defined by

$$
g_{T} \equiv \frac{g_{\theta \theta}}{r^{2}}=\frac{g_{\phi \phi}}{r^{2} \sin ^{2} \theta} .
$$

The two nonvanishing independent components of the extrinsic curvature are $K_{r r}$ and the transverse extrinsic curvature

$$
K_{T} \equiv \frac{K_{\theta \theta}}{r^{2}}=\frac{K_{\phi \phi}}{r^{2} \sin ^{2} \theta} .
$$

The nonvanishing components of $f_{k i j}$ are $f_{r r r}$, the transverse component

$$
f_{r T} \equiv \frac{f_{r \theta \theta}}{r^{2}}=\frac{f_{\theta \theta r}}{2 r^{2}}=\frac{f_{r \phi \phi}}{r^{2} \sin ^{2} \theta}=\frac{f_{\phi \phi r}}{2 r^{2} \sin ^{2} \theta},
$$

and the additional components

$$
\begin{gathered}
f_{r r \theta}=g_{r r} \cot \theta, \\
f_{\theta \theta \theta}=2 r^{2} g_{T} \cot \theta, \\
f_{\theta \phi \phi}=r^{2} g_{T} \sin \theta \cos \theta,
\end{gathered}
$$

$$
f_{\phi \theta \phi}=r^{2} g_{T} \sin \theta \cos \theta .
$$

The evolution equations for these additional components, Eqs. (2.17), are automatically obeyed if the evolution equations for the metric are satisfied. We therefore do not treat these quantities as independent variables, and wherever they appear in the equations we replace them with the appropriate metric components using Eqs. (2.17). Of course, all angular dependence due to these terms drops out.

We therefore take as fundamental variables the six quantities $g_{r r}, g_{T}, K_{r r}, K_{T}, f_{r r r}$, and $f_{r T}$. Using Eqs. (2.4), we obtain the following evolution equations for these variables:

$$
\begin{aligned}
\partial_{t} g_{r r}-\beta^{r} \partial_{r} g_{r r}= & -2 N K_{r r}+2 g_{r r} \partial_{r} \beta^{r} \\
\partial_{t} g_{T}-\beta^{r} \partial_{r} g_{T}= & -2 N K_{T}+2 \frac{\beta^{r}}{r} g_{T} \\
\partial_{t} K_{r r}-\beta^{r} \partial_{r} K_{r r}+\frac{N}{g_{r r}} \partial_{r} f_{r r r}= & N\left[2 f^{r}{ }_{r r}\left(f^{r}{ }_{r r}+\frac{1}{r}-\frac{4 f_{r T}}{g_{T}}\right)-\frac{6}{r^{2}}+K_{r r}\left(2 \frac{K_{T}}{g_{T}}-K_{r}^{r}\right)\right. \\
& \left.-6\left(\frac{f_{r T}}{g_{T}}\right)^{2}-\partial_{r}^{2} \ln \tilde{\alpha}-\left(\partial_{r} \ln \tilde{\alpha}\right)^{2}+\left(\frac{4}{r}-f_{r r}^{r}\right) \partial_{r} \ln \tilde{\alpha}\right]+2 K_{r r} \partial_{r} \beta^{r}+4 \pi N\left(T g_{r r}-2 S_{r r}\right), \\
\partial_{t} K_{T}-\beta^{r} \partial_{r} K_{T}+\frac{N}{g_{r r}} \partial_{r} f_{r T}= & N\left(K_{T} K_{r}^{r}+\frac{1}{r^{2}}-\frac{2 f_{r T}^{2}}{g_{r r} g_{T}}-\frac{f_{r T}}{g_{r r}} \partial_{r} \ln \tilde{\alpha}\right)+\frac{2 \beta^{r}}{r} K_{T}, \\
\partial_{t} f_{r r r}-\beta^{r} \partial_{r} f_{r r r}+N \partial_{r} K_{r r}= & N\left[4 g_{r r} \frac{K_{T}}{g_{T}}\left(3 \frac{f_{r T}}{g_{T}}-f^{r}{ }_{r r}+\frac{2}{r}-\partial_{r} \ln \tilde{\alpha}\right)-K_{r r}\left(10 \frac{f_{r T}}{g_{T}}+f_{r r}^{r}-\frac{2}{r}+\partial_{r} \ln \tilde{\alpha}\right)\right] \\
& +3 f_{r r r} \partial_{r} \beta^{r}+g_{r r} \partial_{r}^{2} \beta^{r}+16 \pi N J_{r} g_{r r}, \\
\partial_{t} f_{r T}-\beta^{r} \partial_{r} f_{r T}+N \partial_{r} K_{T}= & N\left[K_{T}\left(2 \frac{f_{r T}}{g_{T}}-f_{r r}^{r}{ }_{r r}-\partial_{r} \ln \tilde{\alpha}\right)\right]+\left(\partial_{r} \beta^{r}+\frac{2 \beta^{r}}{r}\right) f_{r T} .
\end{aligned}
$$


Here

$$
\widetilde{\alpha} \equiv \alpha r^{2} \sin \theta=\frac{N}{g_{T} \sqrt{g_{r r}}},
$$

and we have explicitly included the terms involving the Lie derivative of the shift vector.

The six fundamental variables obey four constraints that can be obtained from Eqs. (2.8):

$$
\begin{aligned}
\mathcal{C} \equiv & \frac{\partial_{r} f_{r T}}{g_{r r} g_{T}}-\frac{1}{2 r^{2} g_{T}}+\frac{f_{r T}}{g_{r r} g_{T}}\left(\frac{2}{r}+\frac{7 f_{r T}}{2 g_{T}}-f_{r r}^{r}\right) \\
& -\frac{K_{T}}{g_{T}}\left(K_{r}^{r}+\frac{K_{T}}{2 g_{T}}\right)+4 \pi \rho=0,
\end{aligned}
$$

$$
\begin{aligned}
\mathcal{C}_{r} & \equiv \frac{\partial_{r} K_{T}}{g_{T}}+\frac{2 K_{T}}{r g_{T}}-\frac{f_{r T}}{g_{T}}\left(K_{r}^{r}+\frac{K_{T}}{g_{T}}\right)+4 \pi J_{r}=0, \\
\mathcal{C}_{r r r} & \equiv \partial_{r} g_{r r}+\frac{8 g_{r r} f_{r T}}{g_{T}}-2 f_{r r r}=0, \\
\mathcal{C}_{r T} & \equiv \partial_{r} g_{T}+\frac{2 g_{T}}{r}-2 f_{r T}=0 .
\end{aligned}
$$

We do not explicitly solve the constraints during our evolution, but instead we use them as error estimators.

If we are including a Klein-Gordon matter source, we evolve $\Pi$ and $\Phi_{r}$, but not $\phi$. The last two equations of Eqs. (2.12) take the form

$$
\begin{gathered}
\partial_{t} \Pi-\beta^{r} \partial_{r} \Pi+\frac{N}{g_{r r}} \partial_{r} \Phi_{r}=N\left[\Pi\left(\frac{K_{r r}}{g_{r r}}+\frac{2 K_{T}}{g_{T}}\right)-\frac{\Phi_{r}}{g_{r r}}\left(\frac{4 f_{r T}}{g_{T}}-\frac{2}{r}+\partial_{r} \ln \tilde{\alpha}\right)\right], \\
\partial_{t} \Phi_{r}-\beta^{r} \partial_{r} \Phi_{r}+N \partial_{r} \Pi=-N \Pi\left(\frac{f_{r r r}}{g_{r r}}-\frac{2 f_{r T}}{g_{T}}-\frac{2}{r}+\partial_{r} \ln \tilde{\alpha}\right)+\Phi_{r} \partial_{r} \beta^{r} .
\end{gathered}
$$

\section{Boundary conditions}

Boundary conditions are imposed on the above evolution equations (2.18) and (2.21) via characteristic decomposition. Consider a first-order symmetrizable hyperbolic system

$$
\partial_{t} U+A^{i} \partial_{i} U=R,
$$

where $U$ is the vector of variables, $R$ is a vector, and the three $A^{i}$ are matrices. Then for a particular unit vector $\xi_{i}$, the solutions $U_{c}$ to the eigenvalue problem

$$
A^{i} \xi_{i} U_{c}=v_{c} U_{c}
$$

define the characteristic fields normal to the direction $\xi_{i}$, and the eigenvalues $v_{c}$ define the characteristic speeds of these fields. Each of the characteristic fields $U_{c}$ can be thought of as a plane wave solution moving in the direction $\xi_{i}$ with speed $v_{c}$. One is allowed to impose boundary conditions only on characteristic fields that propagate into the computational domain, but not on fields that propagate out of the domain.

For the evolution equations (2.18) and (2.21), the characteristic fields in the radial direction $\left(\xi_{r}=\sqrt{g_{r r}}\right)$ are

$$
\begin{gathered}
U_{r}^{0} \equiv g_{r r} \quad\left(v_{c}=-\beta^{r}\right), \\
U_{t}^{0} \equiv g_{T} \quad\left(v_{c}=-\beta^{r}\right),
\end{gathered}
$$

$$
\begin{gathered}
U_{r}^{ \pm} \equiv K_{r r} \pm \frac{f_{r r r}}{\sqrt{g_{r r}}} \quad\left(v_{c}=-\beta^{r} \pm \tilde{\alpha} g_{T}\right), \\
U_{T}^{ \pm} \equiv K_{T} \pm \frac{f_{r T}}{\sqrt{g_{r r}}} \quad\left(v_{c}=-\beta^{r} \pm \tilde{\alpha} g_{T}\right), \\
U_{\phi}^{ \pm} \equiv \Pi \pm \frac{\Phi_{r}}{\sqrt{g_{r r}}} \quad\left(v_{c}=-\beta^{r} \pm \tilde{\alpha} g_{T}\right) .
\end{gathered}
$$

The characteristic speeds of the metric variables correspond to propagation along the timelike normal to the foliation and the characteristic speeds of the other quantities correspond to propagation along the light cone. Thus, if the inner boundary of our domain moves along a spacelike trajectory, all characteristic speeds are negative (with respect to $r$ ) there, so no boundary conditions need to be imposed. At the outer boundary, boundary conditions are imposed only on those quantities with negative characteristic speeds (usually $U_{r}^{0}, U_{t}^{0}$, $U_{r}^{-}, U_{T}^{-}$, and $U_{\phi}^{-}$).

We have experimented with three types of outer boundary conditions. The first, which we call the freezing boundary condition, is

$$
\partial_{t} U_{c}=0
$$

applied to all incoming characteristic fields $U_{c}$. This corresponds to no incoming radiation at the boundary; however, 
for nonlinear or inhomogeneous problems this is not strictly correct unless the boundary is at infinity. Note that the boundary condition $U_{c}=0$ is insufficient because the incoming characteristic fields contain nonzero longitudinal contributions. This is true even for a simple wave equation with a nonzero source term.

The second outer boundary condition is the Robin condition

$$
\partial_{r}\left[r^{n}\left(U-U_{\infty}\right)\right]=0,
$$

which assumes that $U$ behaves like

$$
U_{\infty}+\frac{\text { const }}{r^{n}}
$$

at large $r$. For a given incoming variable $U_{c}$, appropriate values of the parameters $U_{\infty}$ and $n$ can be found from the analytic representations of the Schwarzschild geometry in the Appendix.

Finally, the constraints (2.20) can be used to derive mixed Neumann-Dirichlet boundary conditions for four of the characteristic fields: Equations (2.20c) and (2.20d) can be used directly as boundary conditions on $U_{r}^{0}$ and $U_{t}^{0}$, and Eqs. (2.20a) and (2.20b) can be combined to yield boundary conditions on $U_{T}^{-}$and $U_{T}^{+}$. We use only three of these boundary conditions - the ones for $U_{r}^{0}, U_{t}^{0}$, and $U_{T}^{-}$-because $U_{T}^{+}$is outgoing at the outer boundary and therefore needs no boundary condition there. Similarly, in three spatial dimensions the constraints can be used to derive 22 relations among the 30 characteristic fields, some of which can be used as boundary conditions on the incoming fields.

We describe our numerical implementation of boundary conditions in Sec. III D.

\section{E. Apparent horizon}

A marginal outer trapped 2-surface is defined by the equation

$$
D_{i} s^{i}+\left(s^{i} s^{j}-g^{i j}\right) K_{i j}=0
$$

where $D_{i}$ is the covariant derivative compatible with the three-metric, and $s^{i}$ is the outward-pointing spatial unit normal of the surface. In spherical symmetry, Eq. (2.28) reduces to

$$
\frac{f_{r T}}{\sqrt{g_{r r}}}-K_{T}=0
$$

The apparent horizon is the outermost surface at which Eq. (2.29) is satisfied. On each time slice, the coordinate radius of the horizon $r_{\text {ah }}$ is located by solving Eq. (2.29) using a standard root-finding algorithm.

If the horizon is to remain at a fixed coordinate radius as the spacetime evolves, the following relation must be obeyed at the horizon:

$$
\frac{\beta^{r}}{\tilde{\alpha} g_{T}}=\frac{1+8 \pi r^{2} g_{T}\left(\rho-J_{r} / \sqrt{g_{r r}}\right)}{1-8 \pi r^{2} g_{T}\left(\rho-J_{r} / \sqrt{g_{r r}}\right)} .
$$

Equation (2.30) can be derived by setting the time derivative of Eq. (2.29) equal to zero and substituting the evolution and constraint equations to eliminate time and spatial derivatives.

\section{F. Gauge conditions}

The question of which gauge conditions one should impose on a numerically generated spacetime is one of the key unsolved problems in numerical relativity. In principle, the coordinate invariance of general relativity allows one to make this choice arbitrarily. However, a poor choice may not only obscure the physics one is searching for in the simulation, but may also allow rapidly growing gauge modes that halt the code altogether.

\section{Algebraic conditions}

The simplest gauge choices we consider here are algebraic ones: we set $\tilde{\alpha}$ and $\beta^{i}$ equal to their analytic values for some parametrization of the Schwarzschild solution in the Appendix that we wish to reproduce numerically. While these gauge conditions are obviously applicable only to test problems, they provide a simplified setting in which to study the properties of our evolution scheme. Such conditions have been used extensively in 3D test problems [25].

Next we consider other algebraic gauge conditions that are independent of a particular analytic solution, but are still of limited generality. For instance, one might require that the radial coordinate remain areal or, in other words, that $g_{T}$ be time independent. Using our variables, this condition can be written

$$
\beta^{r} f_{r T}-\tilde{\alpha} g_{T} \sqrt{g_{r r}} K_{T}=0 .
$$

One might also require that the ingoing coordinate speed of light takes on a prescribed value $c_{-}$:

$$
\tilde{\alpha} g_{T}+\beta^{r}+c_{-}=0 \text {. }
$$

These conditions are not generalizable to two black holes because the first relies on the notion of an areal radial coordinate and the second assumes that a unique "ingoing" direction exists at every point in spacetime. Nevertheless, these and similar gauge conditions have proved useful for studies of single-black-hole spacetimes $[45,46]$. Furthermore, if imposed only at one point, they can be used as boundary conditions on more general elliptic gauge choices, described below.

\section{Elliptic conditions}

We also explore gauge conditions that should be applicable to general spacetimes. For the shift vector, we consider two elliptic equations due to [47]: minimal strain 


$$
\begin{gathered}
\partial_{r}^{2} \beta^{r}+\left(\frac{f_{r r r}}{g_{r r}}-\frac{2 f_{r T}}{g_{T}}\right) \partial_{r} \beta^{r}+\left[\frac{\partial_{r} f_{r r r}}{g_{r r}}-\frac{4 \partial_{r} f_{r T}}{g_{T}}-2\left(\frac{f_{r r r}}{g_{r r}}\right)^{2}+\frac{2 f_{r T}}{g_{T}}\left(\frac{5 f_{r r r}}{g_{r r}}-\frac{f_{r T}}{g_{T}}-\frac{4}{r}\right)\right] \beta^{r} \\
-\frac{K_{r r} g_{T}}{\sqrt{g_{r r}}} \partial_{r} \tilde{\alpha}+\tilde{\alpha} \sqrt{g_{r r}} g_{T}\left[-\frac{\partial_{r} K_{r r}}{g_{r r}}+\frac{2 K_{T} f_{r T}}{\left(g_{T}\right)^{2}}+\frac{K_{r r}}{g_{r r}}\left(\frac{f_{r r r}}{g_{r r}}+\frac{2}{r}-\frac{8 f_{r T}}{g_{T}}\right)\right]=0,
\end{gathered}
$$

which minimizes changes in the three-metric in a global sense, and minimal distortion

$$
\begin{aligned}
\partial_{r}^{2} \beta^{r} & +\left(\frac{f_{r r r}}{g_{r r}}-\frac{2 f_{r T}}{g_{T}}\right) \partial_{r} \beta^{r}+\left[\frac{\partial_{r} f_{r r r}}{g_{r r}}-\frac{5 \partial_{r} f_{r T}}{g_{T}}-2\left(\frac{f_{r r r}}{g_{r r}}\right)^{2}+\frac{f_{r T}}{g_{T}}\left(\frac{11 f_{r r r}}{g_{r r}}-\frac{5 f_{r T}}{g_{T}}-\frac{10}{r}\right)\right] \beta^{r} \\
& +\left(\frac{K_{T}}{g_{T}}-\frac{K_{r r}}{g_{r r}}\right) \sqrt{g_{r r}} g_{T} \partial_{r} \tilde{\alpha}+\tilde{\alpha} \sqrt{g_{r r}} g_{T}\left[-\frac{\partial_{r} K_{r r}}{g_{r r}}+\frac{K_{T}}{g_{T}}\left(\frac{f_{r r r}}{g_{r r}}-\frac{2}{r}\right)+\frac{K_{r r}}{g_{r r}}\left(\frac{f_{r r r}}{g_{r r}}+\frac{2}{r}-\frac{8 f_{r T}}{g_{T}}\right)\right]=0,
\end{aligned}
$$

which similarly minimizes changes of the conformal three-metric.

For the densitized lapse function, we consider the stationary mean curvature condition $\partial_{t} K=0$, which in terms of our variables can be written

$$
\begin{aligned}
\partial_{r}^{2} \tilde{\alpha} & +\left(\frac{f_{r r r}}{g_{r r}}+\frac{2 f_{r T}}{g_{T}}-\frac{4}{r}\right) \partial_{r} \tilde{\alpha}+\tilde{\alpha}\left[\frac{\partial_{r} f_{r r r}}{g_{r r}}+\frac{2 \partial_{r} f_{r T}}{g_{T}}-2\left(\frac{f_{r r r}}{g_{r r}}+\frac{f_{r T}}{g_{T}}\right)\left(\frac{f_{r r r}}{g_{r r}}-\frac{5 f_{r T}}{g_{T}}\right)+\frac{6}{r^{2}}-\frac{2 g_{r r}}{r^{2} g_{T}}-g_{r r}\left(\frac{K_{r r}}{g_{r r}}+\frac{2 K_{T}}{g_{T}}\right)^{2}\right. \\
& \left.-\frac{2 f_{r r r}}{r g_{r r}}+8 \pi S_{r r}-4 \pi g_{r r} T\right]-\frac{\beta^{r} \sqrt{g_{r r}}}{g_{T}}\left[\frac{\partial_{r} K_{r r}}{g_{r r}}+\frac{2 \partial_{r} K_{T}}{g_{T}}+\frac{4 K_{T}}{g_{T}}\left(\frac{1}{r}-\frac{f_{r T}}{g_{T}}\right)+\frac{2 K_{r r}}{g_{r r}}\left(\frac{4 f_{r T}}{g_{T}}-\frac{f_{r r r}}{g_{r r}}\right)\right]=0 .
\end{aligned}
$$

This condition was also discussed by [47], and is best known in the special case $K=0$, when it reduces to the familiar maximal slicing condition. Use of the stationary mean curvature lapse combined with either the minimal strain or minimal distortion shift vectors has been recently encouraged by $[48,49]$.

Each of the elliptic equations (2.33), (2.34), and (2.35) requires two boundary conditions. At the horizon, we either set $\tilde{\alpha}$ and $\beta^{r}$ to prescribed values, or we impose Eqs. (2.30) and (2.32). At the outer boundary, we again can set $\tilde{\alpha}$ and $\beta^{r}$ to prescribed values, we can impose Eqs. (2.31) and (2.32), or we can impose Robin conditions of the form (2.26) on $\widetilde{\alpha}$ and $\beta^{r}$.

\section{G. Mass}

It is useful for diagnostic purposes to compute the mass of the spacetime. In spherical symmetry, the total mass inside an invariant spherical surface labeled by coordinate $r$ is well defined and given by the Misner-Sharp formula [50], which for our variables reads

$$
M_{\mathrm{MS}}(r) \equiv \frac{r \sqrt{g_{T}}}{2}\left[1+\frac{r^{2}}{g_{T}}\left(K_{T}^{2}-\frac{f_{r T}^{2}}{g_{r r}}\right)\right] .
$$

\section{H. Initial data}

\section{Vacuum black holes}

For evolutions of spherically symmetric vacuum black holes, we choose initial data corresponding to one of the time-independent representations of the Schwarzschild ge- ometry listed in the Appendix. For these solutions, all fundamental variables are given by explicit functions of radial coordinate $r$ and black hole mass $M$.

\section{Einstein-Klein-Gordon black holes}

Given arbitrary initial values of the scalar field variables $\Pi$ and $\Phi_{r}$, one must construct initial data for the gravitational field variables such that the Hamiltonian and momentum constraints are satisfied. We solve the constraints via the standard York-Lichnerowicz conformal decomposition [51], using one of the time-independent representations of the Schwarzschild geometry discussed in the Appendix as a background solution.

To perform the decomposition, we first write

$$
\begin{gathered}
K \equiv \frac{K_{r r}}{g_{r r}}+2 \frac{K_{T}}{g_{T}}, \\
A_{r r} \equiv \frac{2}{3}\left(K_{r r}-g_{r r} \frac{K_{T}}{g_{T}}\right), \\
A_{T} \equiv \frac{1}{3}\left(K_{T}-g_{T} \frac{K_{r r}}{g_{r r}}\right),
\end{gathered}
$$

where $A_{r r}$ and $A_{T}$ are the two independent components of the trace-free part of $K_{i j}$ in spherical symmetry. We then assume that 


$$
\begin{aligned}
g_{r r} & \equiv \psi^{4} \bar{g}_{r r}, \\
g_{T} & \equiv \psi^{4} \bar{g}_{T}, \\
A_{r r} & \equiv \psi^{-2} \bar{A}_{r r}, \\
A_{T} & \equiv \psi^{-2} \bar{A}_{T}, \\
f_{r r r} & =\psi^{4}\left(\bar{f}_{r r r}+10 \bar{g}_{r r} \partial_{r} \ln \psi\right), \\
f_{r T} & =\psi^{4}\left(\bar{f}_{r T}+2 \bar{g}_{T} \partial_{r} \ln \psi\right),
\end{aligned}
$$

and we write $\bar{A}_{r r}$ and $\bar{A}_{T}$ as initial guesses $\widetilde{A}_{r r}$ and $\widetilde{A}_{T}$ plus purely longitudinal corrections:

$$
\bar{A}_{r r} \equiv \widetilde{A}_{r r}+\frac{4}{3} \bar{g}_{r r}\left[\partial_{r} v^{r}+v^{r}\left(\frac{\bar{f}_{r r r}}{\bar{g}_{r r}}-5 \frac{\bar{f}_{r T}}{\bar{g}_{T}}\right)\right],
$$

$$
\bar{A}_{T} \equiv \widetilde{A}_{T}-\frac{2}{3} \bar{g}_{T}\left[\partial_{r} v^{r}+v^{r}\left(\frac{\bar{f}_{r r r}}{\bar{g}_{r r}}-5 \frac{\bar{f}_{r T}}{\bar{g}_{T}}\right)\right] .
$$

In Eqs. (2.38) and (2.39), $\psi$ is an unknown conformal factor and $v^{\mu}$ is an unknown vector (with only a radial component in spherical symmetry). The quantities $\bar{g}_{r r}, \bar{g}_{T}, \bar{f}_{r r r}$, and $\bar{f}_{r T}$ are taken as fixed "background" functions, which we obtain from one of the time-independent representations of the Schwarzschild geometry listed in the Appendix. The quantities $K, \widetilde{A}_{r r}$, and $\widetilde{A}_{T}$ are fixed values computed from the extrinsic curvature of the background using Eqs. (2.37). Hence, given a choice of background solution, and given values of $\psi$ and $v^{r}$, one can construct all gravitational variables from Eqs. (2.37)-(2.39).

The quantities $\psi$ and $v^{r}$ are determined by the Hamiltonian and momentum constraints, which take the form

$$
\begin{gathered}
\partial_{r}^{2} \psi+\left(6 \frac{\bar{f}_{r T}}{\bar{g}_{T}}-\frac{\bar{f}_{r r r}}{\bar{g}_{r r}}\right) \partial_{r} \psi-\frac{1}{4} \psi\left[\frac{\bar{g}_{r r}}{\bar{g}_{T} r^{2}}+\frac{\bar{f}_{r T}}{\bar{g}_{T}}\left(2 \frac{\bar{f}_{r r r}}{\bar{g}_{r r}}-7 \frac{\bar{f}_{r T}}{\bar{g}_{T}}-\frac{4}{r}\right)-\frac{2}{\bar{g}_{T}} \partial_{r} \bar{f}_{r T}\right] \\
+\frac{1}{4} \psi^{5}\left(8 \pi \rho-\frac{1}{3} K^{2}\right)+\frac{1}{8} \psi^{-7}\left(\frac{\bar{A}_{r r}^{2}}{\bar{g}_{r r}}+2 \bar{g}_{r r}\left(\frac{\bar{A}_{T}}{\bar{g}_{T}}\right)^{2}\right)=0, \\
\partial_{r}^{2} v^{r}+\left(\frac{\bar{f}_{r r r}}{\bar{g}_{r r}}-2 \frac{\bar{f}_{r T}}{\bar{g}_{T}}\right) \partial_{r} v^{r}+\left[\frac{1}{\bar{g}_{r r}} \partial_{r} \bar{f}_{r r r}-\frac{5}{\bar{g}_{T}} \partial_{r} \bar{f}_{r T}-\left(\frac{\bar{f}_{r r r}}{\bar{g}_{r r}}-5 \frac{\bar{f}_{r T}}{\bar{g}_{T}}\right)\left(2 \frac{\bar{f}_{r r r}}{\bar{g}_{r r}}-\frac{\bar{f}_{r T}}{\bar{g}_{T}}\right)-\frac{10}{r} \frac{\bar{f}_{r T}}{\bar{g}_{T}}\right] v^{r} \\
+\frac{3}{4 \bar{g}_{r r}}\left[\partial_{r} \widetilde{A}_{r r}+2 \widetilde{A}_{r r}\left(5 \frac{\bar{f}_{r T}}{\bar{g}_{T}}-\frac{\bar{f}_{r r r}}{\bar{g}_{r r}}\right)+2 \widetilde{A}_{\theta \theta} \frac{\bar{g}_{r r}}{\bar{g}_{T}} \frac{\bar{f}_{r T}}{\bar{g}_{T}}\right]-\frac{1}{2} \psi^{6} \partial_{r} K-6 \pi J_{r} \psi^{10}=0 .
\end{gathered}
$$

These equations are solved iteratively with $\psi=1$ and $v^{r}=0$ imposed at the inner boundary, and the Robin conditions

$$
\begin{array}{r}
\partial_{r} \psi+c_{\psi}(\psi-1) / r=0, \\
\partial_{r} v^{r}+c_{v} v^{r} / r=0
\end{array}
$$

imposed at the outer boundary. The constants $c_{\psi}$ and $c_{v}$ in Eqs. (2.41) are chosen according to the falloff rates of the background solution. Note that after each iteration of the momentum constraint, one must recompute $\bar{A}_{r r}$ and $\bar{A}_{T}$ for use in the Hamiltonian constraint. Note also that the matter terms appearing in the constraints involve the scalar field. In vacuum and for a background given by one of the expressions in the Appendix, one would obtain $\psi=1$ and $v^{r}=0$.

\section{PSEUDOSPECTRAL COLLOCATION METHODS}

\section{A. Introduction}

Consider a system of $L$ evolution equations of the form

$$
\partial_{t} f^{(l)}=\mathcal{F}^{(l)}\left[\left\{f^{(l)}\right\}\right]
$$

for $1 \leqslant l \leqslant L$, where $\left\{f^{(l)}(\vec{x}, t)\right\}$ is the solution, and $\mathcal{F}^{(l)}\left[\left\{f^{(l)}\right\}\right]$ are (possibly nonlinear) functions of $\left\{f^{(l)}\right\}$ and their spatial derivatives. Approximate each function $f^{(l)}$ of the solution as a finite sum of basis functions $\phi_{k}^{(l)}(\vec{x})$ :

$$
f_{N}^{(l)}(\vec{x}, t)=\sum_{k=0}^{N-1} \widetilde{f}_{k}^{(l)}(t) \phi_{k}^{(l)}(\vec{x})
$$

For smooth functions as $N \rightarrow \infty$ the approximation is exact. Corresponding to the approximate solution $\left\{f_{N}^{(l)}\right\}$ is a residual

$$
R_{N}^{(l)}=\partial_{t} f_{N}^{(l)}-\mathcal{F}^{(l)}\left[\left\{f_{N}^{(l)}\right\}\right],
$$

for each evolution equation.

In PSC the spectral coefficients $\widetilde{f}_{k}^{(l)}(t)$ are determined by demanding that the residuals $R_{N}^{(l)}$ vanish at a fixed set of $N$ collocation points $\vec{x}_{n}$. In other words, it is demanded that the system of differential equations (3.1) be satisfied exactly at the collocation points $\left\{\vec{x}_{n}\right\}$. The choice of the collocation points is intimately related to the choice of basis functions 
used in the approximate solution. In the following subsection, we discuss how they are chosen.

\section{B. Expansion basis and collocation points}

For the remainder of this section, we will restrict ourselves to problems with one spatial dimension. The choice of an expansion basis depends upon the particular problem being solved. For example, the natural expansion basis for a 1D problem with periodic boundary conditions is a Fourier series. For more general boundary conditions, such as the ones we will impose in our black hole evolutions, Chebyshev polynomials are a robust choice for the basis functions. Chebyshev polynomials are defined on the interval

$$
\mathbb{I}=[-1,1]
$$

by

$$
T_{k}(x)=\cos \left(k \cos ^{-1} x\right) .
$$

A function $f$ on $\mathbb{I}$ is approximated as ${ }^{1}$

$$
f_{N}(x, t)=\sum_{k=0}^{N} \tilde{f}_{k}(t) T_{k}(x) .
$$

Note that in order to use this expansion, we must specify a mapping from our physical domain $\left[r_{\min }, r_{\max }\right]$ to $\mathbb{I}$. The simplest choice is a linear mapping, but other choices may work better.

For a Chebyshev expansion, a convenient choice of the collocation points is

$$
x_{n}=\cos \frac{\pi n}{N} \text {. }
$$

At these collocation points, the Chebyshev polynomials satisfy the discrete orthogonality relation

$$
\delta_{j k}=\frac{2}{N \bar{c}_{k}} \sum_{n=0}^{N} \frac{1}{\bar{c}_{n}} T_{j}\left(x_{n}\right) T_{k}\left(x_{n}\right),
$$

where

$$
\bar{c}_{k}= \begin{cases}2, & k=0 \text { or } N, \\ 1, & 1 \leqslant k \leqslant N-1 .\end{cases}
$$

Using the orthogonality relation, the spectral coefficients are given by

$$
\widetilde{f}_{k}=\frac{2}{N \bar{c}_{k}} \sum_{n=0}^{N} \frac{1}{\bar{c}_{n}} f_{N}\left(x_{n}\right) T_{k}\left(x_{n}\right) .
$$

Since

\footnotetext{
${ }^{1}$ For Chebyshev bases the conventional notation is that $k$ runs from 0 to $N$, not $N-1$; thus, there are $N+1$ coefficients and collocation points.
}

$$
T_{k}\left(x_{n}\right)=\cos \frac{\pi k n}{N},
$$

fast cosine transforms can be used to compute Eq. (3.6) at the collocation points and to evaluate Eq. (3.10).

In PSC, the focus is not on the set of spectral coefficients $\left\{\widetilde{f}_{k}(t)\right\}$, but on the equivalent set $\left\{f\left(x_{n}, t\right)\right\}$, the approximate solution evaluated at the collocation points. In particular, the approximate solution to Eq. (3.1) would be given by evolving

$$
\partial_{t} f_{N}^{(l)}\left(x_{n}, t\right)=\mathcal{F}^{(l)}\left(x_{n}, t\right),
$$

for $1 \leqslant l \leqslant L$ and $0 \leqslant n \leqslant N$. Given initial conditions $f^{(l)}(x, 0)$ and appropriate boundary conditions, Eq. (3.12) can be evolved forward in time using the method of lines, described in Sec. III D. Since the focus is on grid-point values, and not the spectral coefficients, it is possible to reuse large amounts of code developed for FD methods.

\section{Computation of derivatives}

The main differences between PSC and FD in evolving Eq. (3.12) are the choice of collocation (grid) points $x_{n}$, how spatial derivatives are computed, and how boundary conditions are imposed. In PSC, spatial derivatives are computed analytically from the series expansion

$$
\frac{\partial f_{N}(x, t)}{\partial x}=\sum_{k=0}^{N} \tilde{f}_{k}(t) \frac{d T_{k}(x)}{d x} .
$$

This derivative can be written as another sum over Chebyshev polynomials

$$
\frac{\partial f_{N}(x, t)}{\partial x}=\sum_{k=0}^{N} \tilde{f}_{k}^{\prime}(t) T_{k}(x),
$$

by using the simple recursion relation

$$
c_{k} \widetilde{f}_{k}^{\prime}(t)=\tilde{f}_{k+2}^{\prime}(t)+2(k+1) \tilde{f}_{k+1}(t),
$$

where

$$
c_{k}= \begin{cases}2, & k=0, \\ 1, & k \geqslant 1 .\end{cases}
$$

Evaluating a derivative requires two fast transforms; the first to compute the spectral coefficients needed in the recursion relation (3.15), the second to evaluate Eq. (3.14).

\section{Time evolution and application of boundary conditions}

We evolve our hyperbolic system using the method of lines. In this method, we cast our system into the form (3.12) and use a standard ordinary differential equation (ODE) solver to integrate the equation in time. For most of the results presented in this paper, we have used a fourth-order explicit Runge-Kutta method. One of the drawbacks of using PSC is that the Chebyshev collocation points (3.7) are clustered near the domain boundaries. This places a more severe 
Courant stability limit $\Delta t \sim O\left(N^{-2}\right)$ on a wave equation than for $\mathrm{FD}$, where $\Delta t \sim \Delta x \sim O\left(N^{-1}\right)$. Because of the superior spatial convergence of PSC, however, this restriction is not as severe as it may seem at first glance. In fact, to retain the accuracy gained by the spatial resolution, it may be necessary to use a time step smaller than that demanded by stability.

One of the advantages of PSC over FD is in how the boundary conditions are applied. In FD, derivatives are approximated by differences of field variables at grid points. The pattern of grid points used must typically be modified at the boundaries of the numerical grid. Consequently, boundary conditions can be difficult to formulate in FD. In PSC, on the other hand, the approximate solution is given over the entire domain. As seen in the previous section, derivatives are computed analytically; therefore nothing special needs to be done to compute the derivative at a boundary. Furthermore, since there are collocation points on the boundary of the domain, the application of boundary conditions is straightforward in PSC. One simply demands that the approximate solution satisfy the exact boundary condition at the boundary collocation point.

The boundary conditions are applied during the time step by modifying $\mathcal{F}^{(l)}\left[\left\{f^{(l)}\right\}\right]$ [cf. Eq. (3.1)] at the boundary points so that the boundary conditions are satisfied. In this paper we are interested in applying boundary conditions on a hyperbolic system of evolution equations. As described in Sec. II D, the solution to a hyperbolic system can be written in terms of characteristic fields that propagate with corresponding characteristic speeds. Physically we know that boundary conditions need only be applied to the incoming characteristic fields.

Therefore, to impose a boundary condition at a domain boundary $x=x_{b}$, we first compute the time derivatives of the characteristic fields $U_{c}\left(x_{b}\right)$ at the boundary. We then apply boundary conditions to the time derivatives of the fields that are propagating into the domain; the remaining fields are untouched. Finally, we reconstruct the time derivatives of the fundamental variables at $x_{b}$ and use these values in the time update. For multi-step time integration algorithms, such as the Runge-Kutta method, the boundary conditions are imposed during each substep. Failure to impose a boundary condition on an incoming field or imposition of a boundary condition on an outgoing field almost always leads to an unstable evolution.

If one wishes to impose a Dirichlet boundary condition $u_{c}\left(x_{b}, t\right)=g(t)$, the easiest method is to rewrite the condition as $\partial_{t} u_{c}\left(x_{b}, t\right)=d g / d t$, and impose the boundary condition on the time derivative as described above. A boundary condition such as Neumann or Robin that involves the spatial derivative of the characteristic field is also enforced during the time update. Computing the time derivatives of the characteristic fields requires spatial derivatives of the fields. At the boundary, one replaces these spatial derivatives with the appropriate values that satisfy the boundary condition.

\section{E. Multiple domains}

In order to use a PSC method for problems of dimension $d$ greater than unity the computational domain must be suf- ficiently simple that it can be mapped to $\mathbb{I}^{d}$ or $\mathbb{I}^{d-2} \times S^{2}$ (where $S^{2}$ are two-spheres). For three dimensions, this typically means a cube, a sphere, or a spherical shell. If the computational domain is more complicated, then it must be decomposed into sub-domains that can each be mapped to one of these domains. For example, in two dimensions an L-shaped region can be decomposed into two adjacent rectangles.

The binary black hole problem will need to be solved using multiple domains. Therefore we test our ability to handle multiple domains on our one-dimensional problems. The use of multiple domains also provides a natural way of making our code run in parallel. We use KeLP [52] to handle communication between multiple domains and for parallelization of our code.

The extension of our method from one domain to multiple domains is straightforward. We evolve each domain independently with communication done only at the boundaries. At the domain boundaries we compute the time derivatives of the characteristic fields in each domain. We then replace the time derivatives of the incoming characteristic fields at the boundary with the time derivatives of the outgoing characteristic fields of the neighboring domain. If there is no neighboring domain at a particular boundary, the external boundary condition is applied as described in Sec. III D.

\section{F. Solving elliptic equations}

In addition to evolving our hyperbolic system of evolution equations (2.4), we may need to solve elliptic equations in order to construct initial data for the Einstein-Klein-Gordon system or to enforce elliptic gauge conditions. Consider a linear elliptic equation of the form

$$
\mathcal{L}(u(x))=f(x),
$$

where $u(x)$ is the solution we are seeking. This can be cast as a matrix problem where, unlike for FD, the matrix corresponding to the linear operator $\mathcal{L}$ is full. In $1 \mathrm{D}$ we solve this matrix equation directly, but for higher-dimensional problems, it will be more efficient to use an iterative method.

A nonlinear elliptic equation such as the Hamiltonian constraint can be solved either by the methods described in [40] or by linearizing the nonlinear system and iterating the linearized equations until a solution is found. The latter method is employed in the work described here.

\section{G. Filtering}

The errors in a spectral method are dominated by two types of terms of roughly equal magnitude. Truncation error arises from the neglect of the high-frequency terms that are not retained in the truncated series. Aliasing error occurs because each neglected high-frequency mode is indistinguishable from some retained lower-frequency mode when sampled only at the collocation points; for example, the functions $\sin (\pi x / 5)$ and $\sin (-9 \pi x / 5)$ take the same values on a grid of $N$ points $x \in\{0,1,2, \ldots, N-1\}$. Because of aliasing 
error, power in the high-frequency mode, instead of being completely neglected, ends up contributing to the lowerfrequency mode.

When solving a nonlinear system of equations it becomes important to control the aliasing error. This can be done by filtering the high-frequency modes of the retained series. For quadratic nonlinearities it is sufficient to zero the top third of the spectral coefficients to eliminate aliasing [33]. In our 1D evolutions, we have found it necessary to filter only gauge variables that are computed from an elliptic equation. In effect we are smoothing the solutions to the elliptic gauge equations to eliminate high-frequency noise. Our preliminary investigations suggest that more extensive filtering may be required to produce stable evolutions in 3D.

\section{NUMERICAL RESULTS}

\section{A. Schwarzschild black hole}

In this section we evolve a time-independent slicing of a Schwarzschild black hole. We begin our numerical evolutions with initial data corresponding to one of the slicings given in the Appendix. If the evolution equations are integrated exactly, the solution will remain time-independent. We can test the convergence of our method by measuring the deviation of the solution from the initial data at a given coordinate time, or by measuring the constraint quantities (2.20), which are zero for the exact solution. For all evolutions, the interior of the hole is excised, and no boundary condition is applied at the inner boundary because all characteristic fields are outgoing (off the domain) there. In Tables I and II we list the input parameters and the results for selected evolutions.

\section{Analytic gauge conditions}

The simplest gauge treatment is to fix the gauge variables $\tilde{\alpha}$ and $\beta^{r}$ to their initial values during the entire evolution. For example, one can begin the evolution with Kerr-Schild initial data (A1) and set $\tilde{\alpha}$ and $\beta^{r}$ according to the analytic expressions (A1c)-(A1d) for all time. In Figs. 1 and 2 we plot the norm of the Hamiltonian constraint and the deviation of $g_{r r}$ from the analytic solution versus time for such an evolution (run 1 from Table I). Each plot shows results for several spatial resolutions $N_{r}$ run at a fixed time resolution $\Delta t=0.007 M$. The features near $t=10 M$ correspond to a small error pulse that begins at the outer boundary at $t=0$, grows like $r^{-2}$ as it propagates inwards, and eventually falls into the hole. After several crossing times, the evolution settles into a steady state that converges to the analytic solution as one increases the spatial resolution. We end the evolutions at $t=11000 \mathrm{M}$ even though they clearly would have proceeded further. The convergence rate is exponential until machine roundoff errors dominate, as illustrated in Fig. 3. Repeating the evolutions shown in Figs. 1-3 for PainlevéGullstrand initial data (run 6 from Table I) yields similar results.

In Fig. 4 we show the norm of the Hamiltonian constraint for run 11 of Table I. This evolution is identical to run 1 except the initial data, as well as the values of $\tilde{\alpha}$ and $\beta^{r}$ for
TABLE I. Input parameters for selected evolutions with analytic gauge conditions. For each evolution we list the initial data type (ID), the domain boundaries in units of $r / M$ (Bounds), the outer boundary condition on $U_{T}^{-}(\mathrm{OBT})$, the outer boundary condition on both $U_{r}^{0}$ and $U_{t}^{0}(\mathrm{OB} 0)$, the time stepping algorithm (TS), and the result of the evolution (Res).

\begin{tabular}{|c|c|c|c|c|c|c|c|}
\hline \multirow{2}{*}{$\frac{\text { Run }}{1}$} & \multirow{2}{*}{$\frac{\mathrm{ID}^{\mathrm{a}}}{\mathrm{KS}}$} & \multicolumn{2}{|c|}{ Bounds } & \multirow{2}{*}{$\frac{\mathrm{OBT}^{\mathrm{b}}}{\mathrm{F}}$} & \multirow{2}{*}{$\frac{\mathrm{OB} 0^{\mathrm{b}}}{\mathrm{F}}$} & \multirow{2}{*}{$\frac{\mathrm{TS}^{\mathrm{c}}}{\mathrm{R} 4}$} & \multirow{2}{*}{$\frac{\operatorname{Res}^{\mathrm{d}}}{\mathrm{Stb}}$} \\
\hline & & 1.9 & 11.9 & & & & \\
\hline 2 & $\mathrm{KS}$ & 1.9 & 11.9 & C & F & $\mathrm{R} 4$ & Stb \\
\hline 3 & $\mathrm{KS}$ & 1.9 & 11.9 & $\mathrm{~F}$ & $\mathrm{C}$ & $\mathrm{R} 4$ & Stb \\
\hline 4 & $\mathrm{KS}$ & 1.9 & 11.9 & $\mathrm{C}$ & $\mathrm{C}$ & $\mathrm{R} 4$ & Stb \\
\hline 5 & $\mathrm{KS}$ & 1.75 & 120 & $\mathrm{C}$ & $\mathrm{C}$ & $\mathrm{R} 4$ & Stb \\
\hline 6 & PG & 1.9 & 11.9 & $\mathrm{~F}$ & $\mathrm{~F}$ & $\mathrm{R} 4$ & Stb \\
\hline 7 & PG & 1.9 & 11.9 & $\mathrm{C}$ & $\mathrm{F}$ & $\mathrm{R} 4$ & Stb \\
\hline 8 & PG & 1.9 & 11.9 & $\mathrm{~F}$ & $\mathrm{C}$ & $\mathrm{R} 4$ & LG \\
\hline 9 & PG & 1.9 & 11.9 & $\mathrm{C}$ & $\mathrm{C}$ & $\mathrm{R} 4$ & LG \\
\hline 10 & PG & 1.75 & 120 & $\mathrm{C}$ & $\mathrm{C}$ & $\mathrm{R} 4$ & Stb \\
\hline 11 & $\mathrm{H}$ & 1.9 & 11.9 & $\mathrm{~F}$ & $\mathrm{~F}$ & $\mathrm{R} 4$ & Exp \\
\hline 12 & $\mathrm{H}$ & 1.9 & 3.9 & $\mathrm{~F}$ & $\mathrm{~F}$ & $\mathrm{R} 4$ & Stb \\
\hline 13 & $\mathrm{H}$ & 1.9 & 11.9 & $\mathrm{C}$ & $\mathrm{F}$ & $\mathrm{R} 4$ & Exp \\
\hline 14 & $\mathrm{H}$ & 1.9 & 11.9 & $\mathrm{~F}$ & $\mathrm{C}$ & $\mathrm{R} 4$ & Exp \\
\hline 15 & $\mathrm{H}$ & 1.9 & 11.9 & $\mathrm{C}$ & $\mathrm{C}$ & $\mathrm{R} 4$ & QG \\
\hline 16 & $\mathrm{H}$ & 1.9 & 11.9 & $\mathrm{C}$ & $\mathrm{C}$ & $\mathrm{BE}$ & QG \\
\hline 17 & $\mathrm{H}$ & 1.75 & 120 & $\mathrm{C}$ & $\mathrm{C}$ & $\mathrm{R} 4$ & LG \\
\hline 18 & $\mathrm{FH}$ & 0.9 & 10.9 & $\mathrm{~F}$ & $\mathrm{~F}$ & $\mathrm{R} 4$ & Exp \\
\hline 19 & $\mathrm{FH}$ & 0.9 & 10.9 & $\mathrm{~F}$ & $\mathrm{C}$ & $\mathrm{R} 4$ & Exp \\
\hline 20 & $\mathrm{FH}$ & 0.9 & 10.9 & $\mathrm{C}$ & $\mathrm{F}$ & $\mathrm{R} 4$ & Exp \\
\hline 21 & $\mathrm{FH}$ & 0.9 & 10.9 & $\mathrm{C}$ & $\mathrm{C}$ & $\mathrm{R} 4$ & QG \\
\hline 22 & $\mathrm{FH}$ & 0.9 & 120 & $\mathrm{C}$ & $\mathrm{C}$ & R4 & LG \\
\hline 23 & $\mathrm{FH}$ & 0.9 & 6.9 & $\mathrm{~F}$ & $\mathrm{~F}$ & $\mathrm{BE}$ & Stb \\
\hline 24 & FH & 0.9 & 7.9 & $\mathrm{~F}$ & $\mathrm{~F}$ & $\mathrm{BE}$ & Exp \\
\hline
\end{tabular}

a PG, Painlevé-Gullstrand; KS, Kerr-Schild; H, harmonic time; FH, fully harmonic.

${ }^{\mathrm{b}} \mathrm{F}$, freezing; C, constraint.

${ }^{\mathrm{c}} \mathrm{R} 4$, 4th-order Runge-Kutta; BE, backward Euler.

${ }^{\mathrm{d}}$ Stb, stable; Exp, exponential growth; LG, linearly growing gauge mode; QG, quadratically growing gauge mode.

all time, correspond to a time-independent harmonic slice of the Schwarzschild geometry (A3). Rather than settling to a steady state, the numerical solution grows exponentially at late times, eventually crashing the code. This is caused by a combination of high-frequency numerical instabilities, rapidly growing gauge modes, and rapidly growing constraint violating modes, all of which can be suppressed by appropriate changes in the evolution algorithm, as described below. Evolutions of fully harmonic initial data (A4) behave similarly. It is not known why these instabilities are absent in evolutions of Kerr-Schild and Painlevé-Gullstrand initial data. However, the dependence of stability on the choice of initial data should not be too surprising if one thinks of the initial data as a background solution and the numerical evolution as a perturbation on this background: in general, modifying the background solution can change the stability of perturbations.

For the evolutions shown in Fig. 4, freezing boundary conditions (2.25) are imposed on the incoming characteristic 
TABLE II. Input parameters for selected evolutions using elliptic gauge conditions. For each evolution we list the initial data type (ID), the outer boundary condition on $U_{T}^{-}$(OBT), the outer boundary condition on both $U_{r}^{0}$ and $U_{t}^{0}(\mathrm{OB} 0)$, the inner and outer boundary conditions on the stationary mean curvature densitized lapse (LapseBC), the shift equation used (Shift), the outer boundary condition on the shift (ShiftOB), the time stepping algorithm (TS), and the result of the evolution (Res). The domain is $(0.9 M, 10.9 M)$ for fully harmonic initial data and $(1.9 M, 11.9 M)$ for all other cases. The inner boundary condition on the shift is given by Eq. (2.30), and all inner boundary conditions on gauge variables are imposed at the current location of the apparent horizon.

\begin{tabular}{|c|c|c|c|c|c|c|c|c|c|}
\hline Run & $\mathrm{ID}^{\mathrm{a}}$ & $\mathrm{OBT}^{\mathrm{b}}$ & $\mathrm{OB} 0^{\mathrm{b}}$ & Lapse & $\mathrm{BC}^{\mathrm{c}}$ & Shift $^{\mathrm{d}}$ & ShiftOB & $\mathrm{TS}^{\mathrm{e}}$ & $\operatorname{Res}^{f}$ \\
\hline 25 & $\mathrm{KS}$ & $\mathrm{F}$ & $\mathrm{F}$ & $c(1)$ & $c(1)$ & MD & $\partial_{t} g_{T}=0$ & R4 & Exp \\
\hline 26 & $\mathrm{KS}$ & $\mathrm{F}$ & $\mathrm{F}$ & $\mathrm{c}(1)$ & $c(1)$ & MS & $\partial_{t} g_{T}=0$ & $\mathrm{R} 4$ & Exp \\
\hline 27 & KS & $\mathrm{C}$ & F & $\mathrm{c}(1)$ & $c(1)$ & MS & $\partial_{t} g_{T}=0$ & R4 & LG \\
\hline 28 & $\mathrm{KS}$ & $\mathrm{F}$ & $\mathrm{C}$ & $\mathrm{c}(1)$ & $c(1)$ & MS & $\partial_{t} g_{T}=0$ & $\mathrm{R} 4$ & Exp \\
\hline 29 & $\mathrm{KS}$ & $\mathrm{C}$ & $\mathrm{C}$ & $c(1)$ & $c(1)$ & MS & $\partial_{t} g_{T}=0$ & $\mathrm{R} 4$ & LG \\
\hline 30 & $\mathrm{KS}$ & $\mathrm{C}$ & $\mathrm{C}$ & $\mathrm{c}(1)$ & $c(1)$ & MD & $\partial_{t} g_{T}=0$ & $\mathrm{R} 4$ & LG \\
\hline 31 & $\mathrm{KS}$ & $\mathrm{C}$ & $\mathrm{C}$ & $c(1)$ & $c(1)$ & MS & $\partial_{t} g_{T}=0$ & $\mathrm{BE}$ & LG \\
\hline 32 & PG & $\mathrm{F}$ & F & $c(2)$ & $\mathrm{F}$ & MS & Robin & $\mathrm{R} 4$ & Exp \\
\hline 33 & PG & $\mathrm{C}$ & $\mathrm{F}$ & $c(2)$ & $\mathrm{F}$ & MS & Robin & $\mathrm{R} 4$ & Exp \\
\hline 34 & PG & $\mathrm{F}$ & $\mathrm{C}$ & $c(2)$ & $\mathrm{F}$ & MS & Robin & R4 & LG \\
\hline 35 & PG & $\mathrm{C}$ & $\mathrm{C}$ & $c(2)$ & $\mathrm{F}$ & MS & Robin & $\mathrm{R} 4$ & LG \\
\hline 36 & PG & $\mathrm{C}$ & $\mathrm{C}$ & $\mathrm{c}(2)$ & $\mathrm{F}$ & MS & Robin & $\mathrm{BE}$ & LG \\
\hline 37 & $\mathrm{H}$ & $\mathrm{C}$ & $\mathrm{C}$ & $c(1 / 2)$ & $\mathrm{F}$ & MS & $\partial_{t} g_{T}=0$ & $\mathrm{BE}$ & LG \\
\hline 38 & $\mathrm{H}$ & $\mathrm{C}$ & $\mathrm{C}$ & $c(1 / 2)$ & $\mathrm{F}$ & MS & $\partial_{t} g_{T}=0$ & R4 & LG \\
\hline 39 & FH & $\mathrm{C}$ & $\mathrm{C}$ & $c(1 / 2)$ & $\mathrm{F}$ & MS & $\partial_{t} g_{T}=0$ & $\mathrm{BE}$ & LG \\
\hline
\end{tabular}

${ }^{a}$ PG, Painlevé-Gullstrand; KS, Kerr-Schild; H, harmonic time; FH, fully harmonic.

${ }^{\mathrm{b}} \mathrm{F}$, freezing; $\mathrm{C}$, constraint.

${ }^{\mathrm{c}} \mathrm{F}$, freezing; $\mathrm{c}(x)$ : Equation (2.32) with $c_{-}=-x$.

${ }^{\mathrm{d}} \mathrm{MS}$, minimal strain; MD, minimal distortion.

${ }^{\mathrm{e}} \mathrm{R} 4$, 4th-order Runge-Kutta; BE, backward Euler.

${ }^{\mathrm{f}}$ Exp, exponential growth; LG, linearly growing gauge mode.

fields $U_{r}^{0}, U_{t}^{0}, U_{r}^{-}$, and $U_{T}^{-}$. One can suppress the constraint-violating modes seen in Fig. 4 by replacing the freezing boundary conditions on $U_{r}^{0}, U_{t}^{0}$, and $U_{T}^{-}$with constraint boundary conditions as discussed in Sec. II D. The resulting evolutions are shown in Figs. 5 and 6. Except for the evolution with $N_{r}=32$ discussed below, the Hamiltonian constraint $\mathcal{C}$ settles to a steady state that converges exponentially to zero. The same is true for the other three constraints $\mathcal{C}_{r T}, \mathcal{C}_{r r r}$, and $\mathcal{C}_{r}$. However, the metric quantities and other fundamental variables grow approximately quadratically with time, eventually causing the simulations to terminate. Because the constraints remain satisfied, we attribute this quadratic growth to a gauge mode.

The $N_{r}=32$ case shown in Figs. 5 and 6 suffers from high-frequency noise that grows exponentially in time. We have experimented with various methods of damping this noise, including filtering the fundamental variables after each time step and adding numerical dissipation terms to the equations. However, we have obtained best results by changing our fourth order Runge-Kutta time-stepping algorithm to an implicit backwards Euler scheme, which is much more dis-

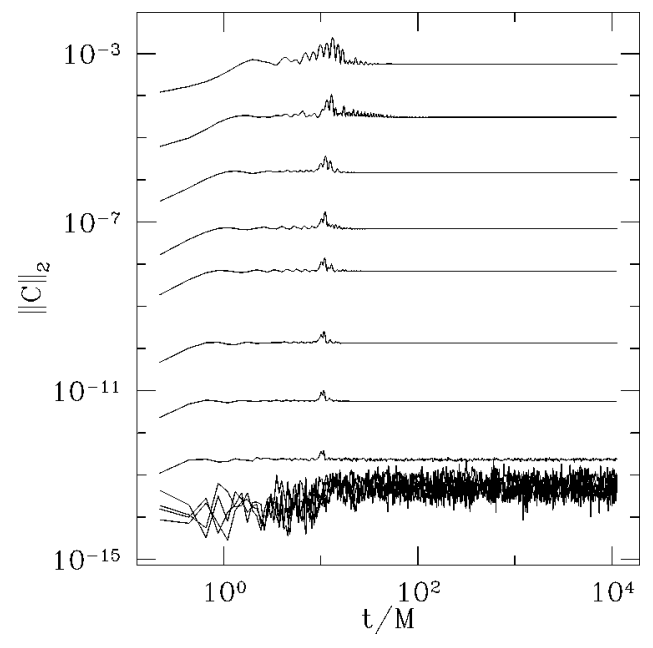

FIG. 1. Long-term stability of the evolution of Kerr-Schild initial data, run 1 from Table I. Plotted is the $l_{2}$ norm of the Hamiltonian constraint (2.20a) in units of $M^{-2}$ as a function of time for several spatial resolutions. The number of spectral coefficients $N_{r}$ for each plot, starting at the top, is $12,16,20,24,27,32,36,40$, $45,48,54$, and 60 .

sipative. Figures 7 and 8 show the results of this modification. The evolution now satisfies the constraints at late times for sufficiently fine resolution, but still suffers from a quadratically growing gauge mode that causes the coarser resolution runs to crash. This gauge mode can be suppressed by applying active gauge conditions, as shown in Sec. IV A 2 below. Evolutions of fully harmonic initial data (A4) produce results similar to those shown in Figs. 4-8.

We note that even with analytic gauge conditions and freezing outer boundary conditions, evolutions of harmonic and fully harmonic initial data such as those shown in Fig. 4 become stable when the outer boundary is moved sufficiently close to the black hole (see runs 12 and 23). A similar dependence on the outer boundary location has also been reported by others $[21,27]$. A possible explanation for this is discussed briefly in [21]: For a nonzero shift vector, any

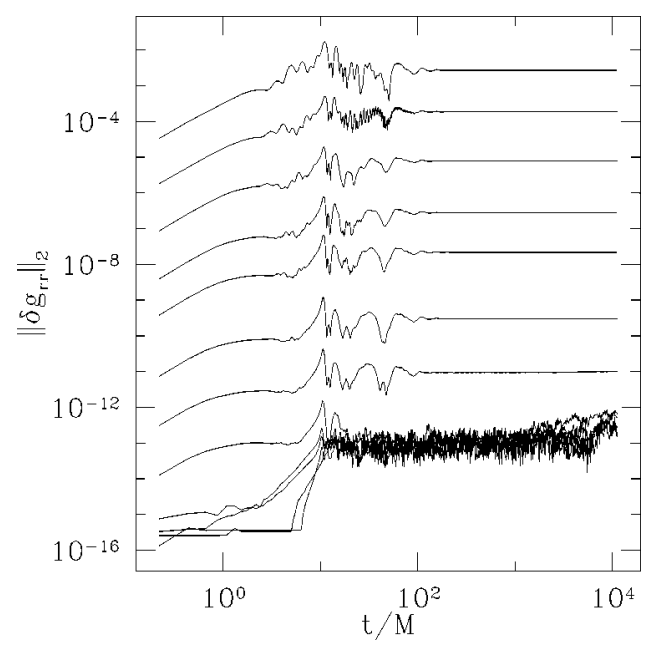

FIG. 2. Norm of the error in $g_{r r}$ as a function of time for the same evolutions shown in Fig. 1. 

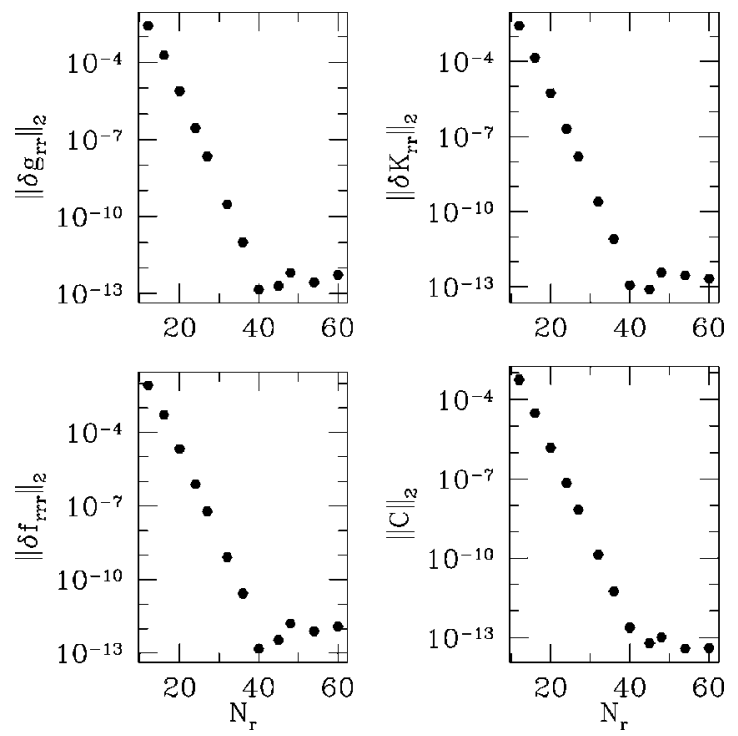

FIG. 3. Norms of the Hamiltonian constraint and errors in selected fundamental variables plotted as a function of the number of spectral coefficients $N_{r}$ at $t=11000 M$ for the evolutions shown in Fig. 1. The quantities $\delta K_{r r}$ and $\delta f_{r r r}$ are measured in units of $M^{-1}$. The errors decrease exponentially with $N_{r}$.

unstable zero-speed modes present in the solution will propagate inward from the outer boundary with speed $-\beta^{r}$, growing as they propagate. If the domain is sufficiently small, these modes do not have time to grow appreciably before they are swallowed by the horizon. As discussed previously, we find that constraint boundary conditions suppress exponentially growing modes, and thus allow evolutions with a larger outer boundary radius (runs 5, 10, 17 and 22).

\section{Elliptic gauge conditions}

Although choosing a time-independent $\beta^{r}$ and $\tilde{\alpha}$ is the simplest gauge condition to implement, for an evolving nu-

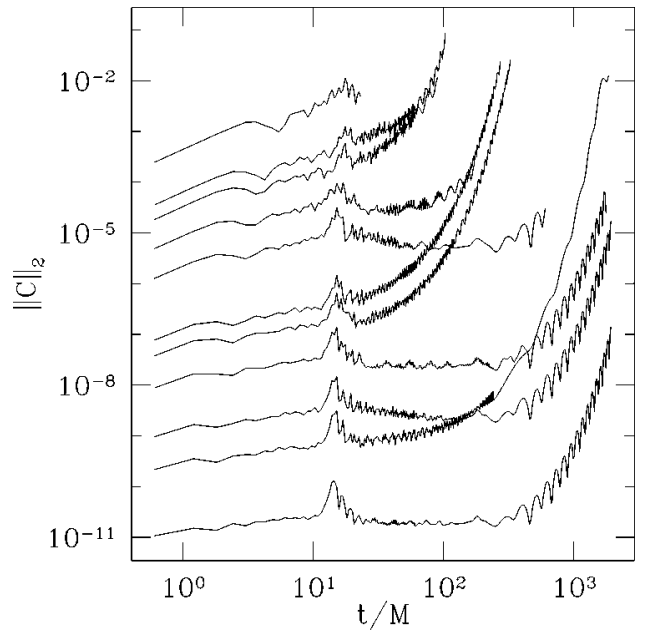

FIG. 4. Norm of the Hamiltonian constraint (2.20a) as a function of time for several spatial resolutions for evolutions of harmonic initial data, run 11 of Table I. The number of spectral coefficients $N_{r}$ for each plot, starting at the top, is $12,15,16,18,20$, $24,25,27,30,32$, and 36 .

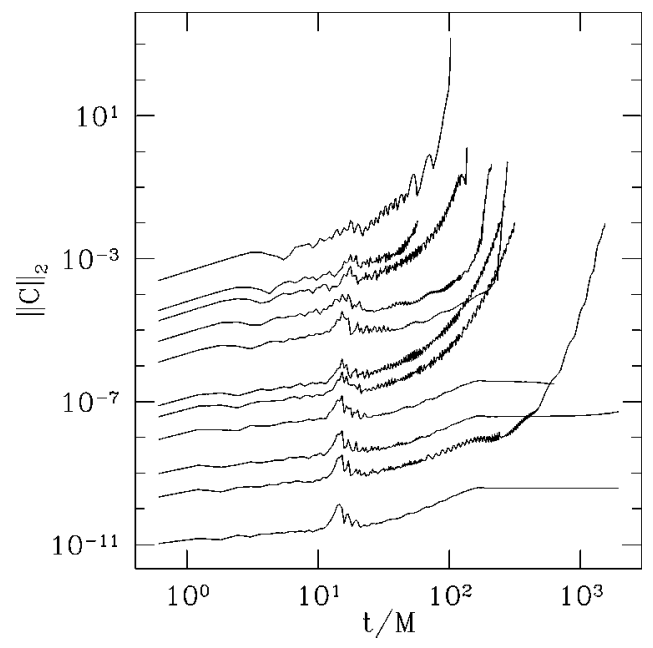

FIG. 5. Norm of the Hamiltonian constraint (2.20a) as a function of time for several spatial resolutions for evolutions of harmonic initial data, run 15 of Table I. Constraint-based outer boundary conditions are imposed on $U_{r}^{0}, U_{t}^{0}$, and $U_{T}^{-}$. Resolutions are the same as in Fig. 4.

merical solution such a choice does not actively enforce any particular coordinate condition. In fact, it is remarkable that many of the cases discussed in Sec. IV A 1 remain stable when the coordinates experience small deviations from the exact solution. For more than one black hole in three spatial dimensions, one will almost certainly need general gauge conditions designed to prevent large changes in the numerical solution of a stationary or quasi-stationary spacetime.

Figure 9 shows the norm of the Hamiltonian constraint for an evolution of Painlevé-Gullstrand initial data. The gauge variables $\beta^{r}$ and $\tilde{\alpha}$ are computed by solving the minimal strain and stationary mean curvature equations (2.33) and (2.35) after each time step. These elliptic equations require boundary conditions. We impose Eqs. (2.30) and (2.32) at the current location of the horizon, which we recompute after every time step. For Eq. (2.32) we choose $c_{-}=-2$, which is the value of $c_{-}$at the horizon for the analytic solution (A2).

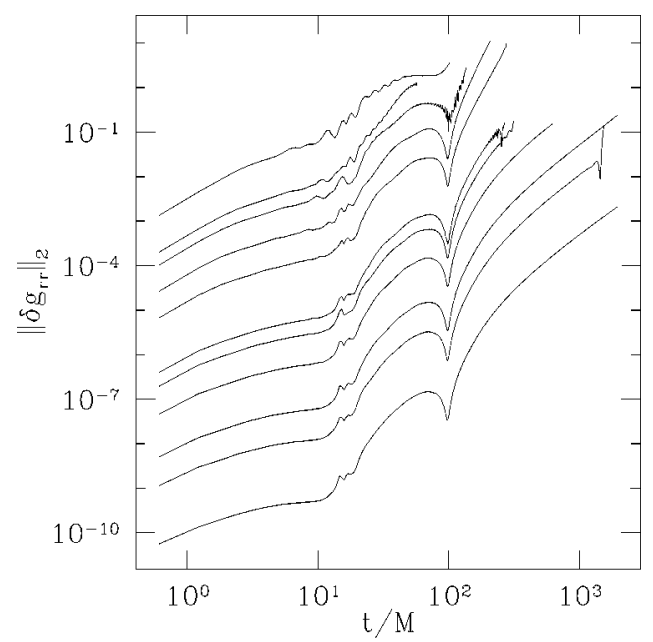

FIG. 6. Error in $g_{r r}$ versus time for the same evolutions shown in Fig. 5. The growth is quadratic in $t$ at late times. 


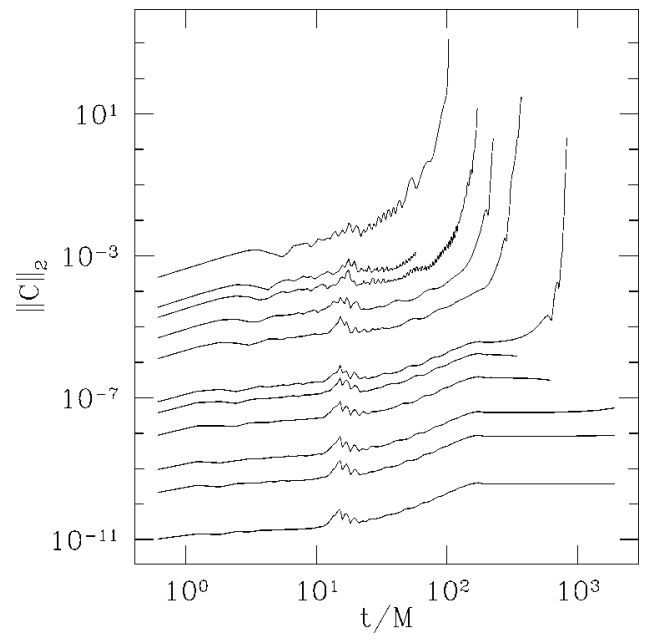

FIG. 7. Norm of the Hamiltonian constraint (2.20a) as a function of time for several spatial resolutions for evolutions of harmonic initial data, run 16 of Table I. The evolutions are identical to those in Fig. 5 except a backwards Euler time stepping scheme is used.

At the outer boundary, we set $\tilde{\alpha}=1$ and we impose a Robin condition (2.26) on $\beta^{r}$ with $\beta_{\infty}^{r}=0, n=1 / 2$. As seen in the figure, the evolution remains stable and convergent. To achieve stability, we find it necessary to apply a simple $2 / 3$ cutoff filter to $\tilde{\alpha}$ and $\beta^{r}$ each time they are computed, and to impose constraint boundary conditions on $U_{r}^{0}$ and $U_{t}^{0}$ (but not on $U_{T}^{-}$). Figure 10 shows the error in $g_{r r}$ for the same evolution. For the highest resolution, one can see a linearlygrowing gauge mode. Although modes that grow linearly will eventually terminate a simulation, they pose no difficulty for long-term evolutions because a much longer run time can be achieved by a modest increase in resolution.

Similar results for the case of harmonic initial data are shown in Figs. 11 and 12. The evolution is stable and convergent, and the rapidly growing gauge mode that terminated the simulation in the case of time-independent $\beta^{r}$ and $\tilde{\alpha}$

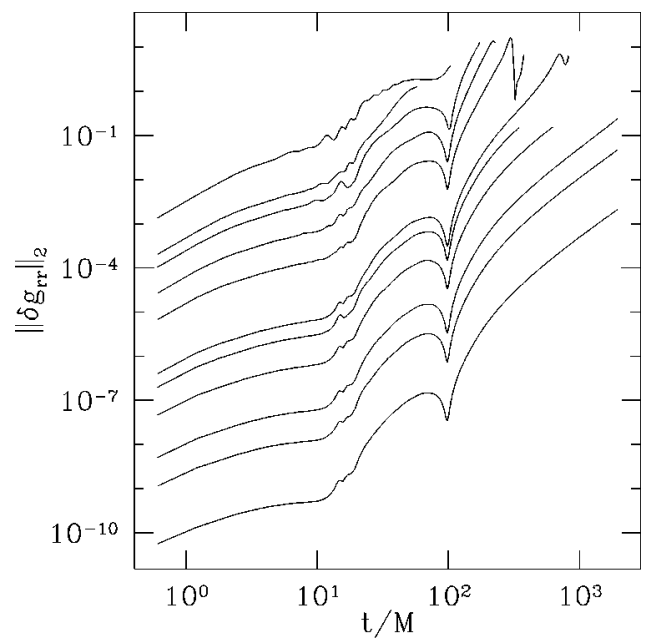

FIG. 8. Error in $g_{r r}$ versus time for the same evolutions shown in Fig. 7. The growth is quadratic in $t$ at late times.

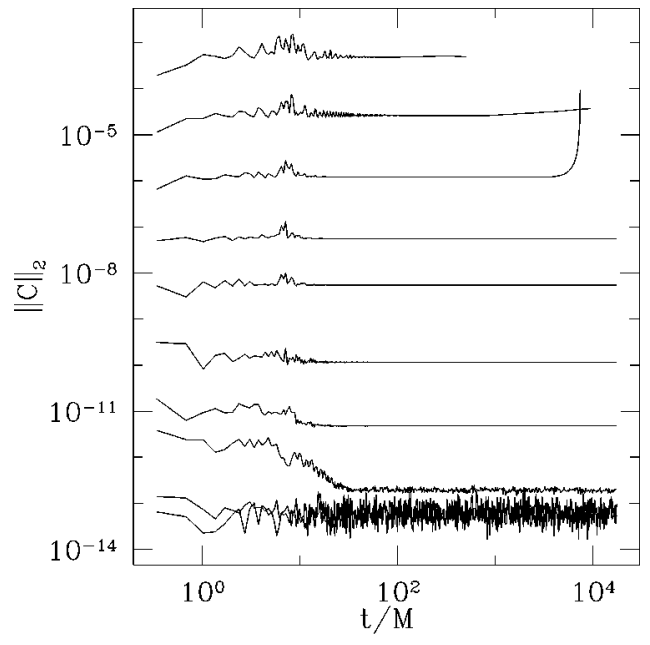

FIG. 9. Norm of the Hamiltonian constraint (2.20a) as a function of time for several spatial resolutions $N_{r}$ for evolutions of Painlevé-Gullstrand initial data using elliptic gauge conditions, run 35 of Table II. The number of spectral coefficients $N_{r}$ for each plot, starting at the top, is $12,16,20,24,27,32,36,40,45$, and 48.

(Sec. IV A 1, Fig. 8) now grows only linearly with time. As in the case shown in Fig. 8, we use a backwards Euler scheme for time evolution. For a fourth-order Runge-Kutta time discretization, results are similar except the evolutions with $N_{r}=25,30$, and 32 are unstable.

\section{B. Black hole plus scalar wave}

In this subsection, we add dynamics to our spherically symmetric spacetime by including a Klein-Gordon scalar field as a matter source. As discussed in Sec. II H, to construct initial data we first choose an arbitrary background spacetime and arbitrary values for $\Pi$ and $\Phi_{r}$, and then we solve the constraints.

For the evolutions described here, the scalar field $\Pi$ is initially a Gaussian centered at $r=20 M$ with a width of $5 M$

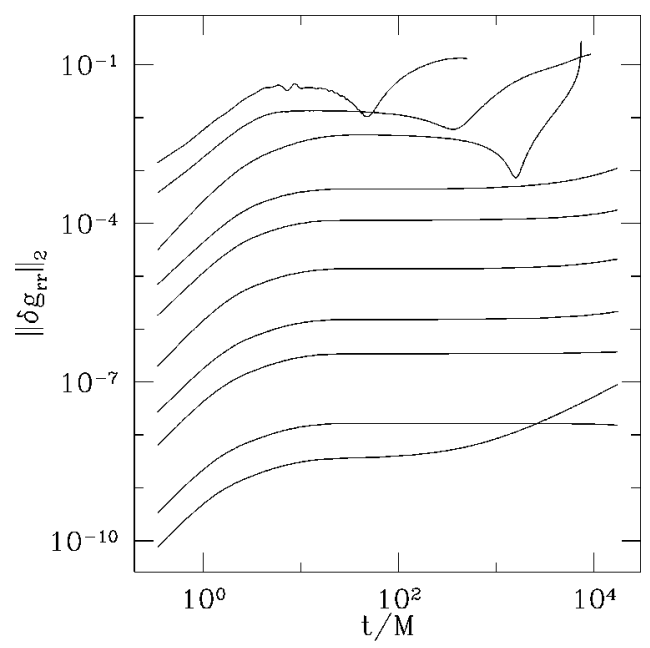

FIG. 10. Error in $g_{r r}$ versus time for the same evolutions shown in Fig. 9. For the highest resolution, the growth is only linear in $t$ at late times. 


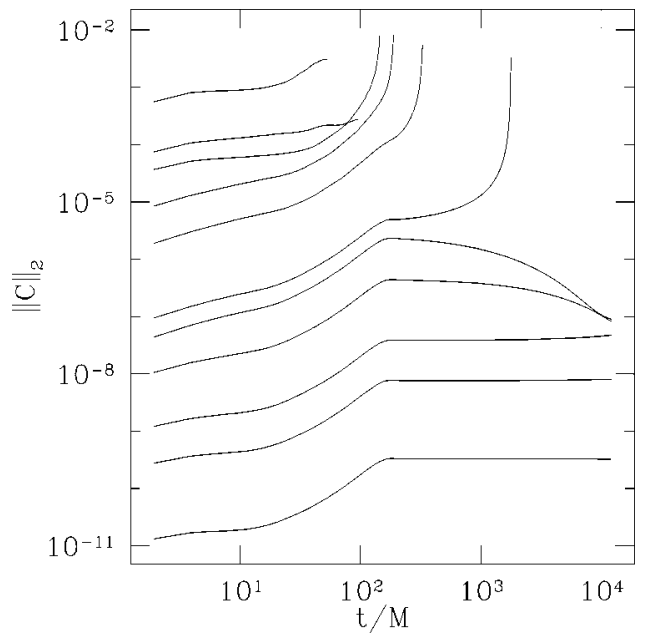

FIG. 11. Norm of the Hamiltonian constraint (2.20a) as a function of time for several spatial resolutions for evolutions of harmonic initial data using elliptic gauge conditions, run 37 of Table II. Resolutions are the same as in Fig. 4.

and an amplitude of $0.02 / M$, and $\Phi_{r}$ is zero everywhere. The outer boundary is located at $r=120 \mathrm{M}$ and the inner boundary is at $1.75 M$. Here $M$ is the mass of the background solution, which is different than the actual mass of the spacetime. The background is chosen to be the Kerr-Schild solution (A1), and the constants in the Robin boundary conditions (2.41) on the constraint variables are $c_{\psi}=c_{v}=1$.

During the evolution, we choose analytic gauge conditions and a fourth-order Runge-Kutta time stepping algorithm. At the outer boundary we apply freezing boundary conditions (2.25) to $U_{\phi}^{-}$and $U_{r}^{-}$, and constraint boundary conditions to $U_{r}^{0}, U_{t}^{0}$ and $U_{T}^{-}$. There is no boundary condition imposed at the inner boundary.

To demonstrate our ability to handle multiple domains, for this evolution we cover the entire domain with 8 equalsized abutting subdomains, each using 45 spectral coefficients. At each domain boundary, the incoming characteristic quantities in each domain are set equal to the corresponding

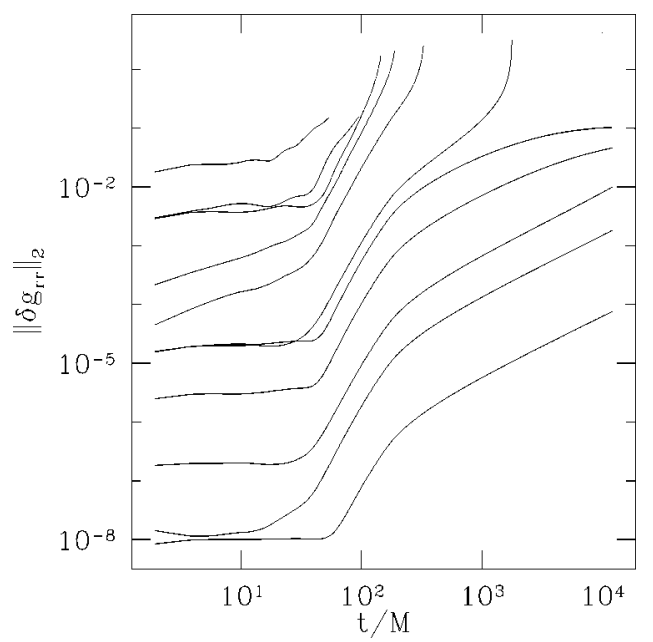

FIG. 12. Error in $g_{r r}$ versus time for the same evolutions shown in Fig. 11. The growth is only linear in $t$ at late times.

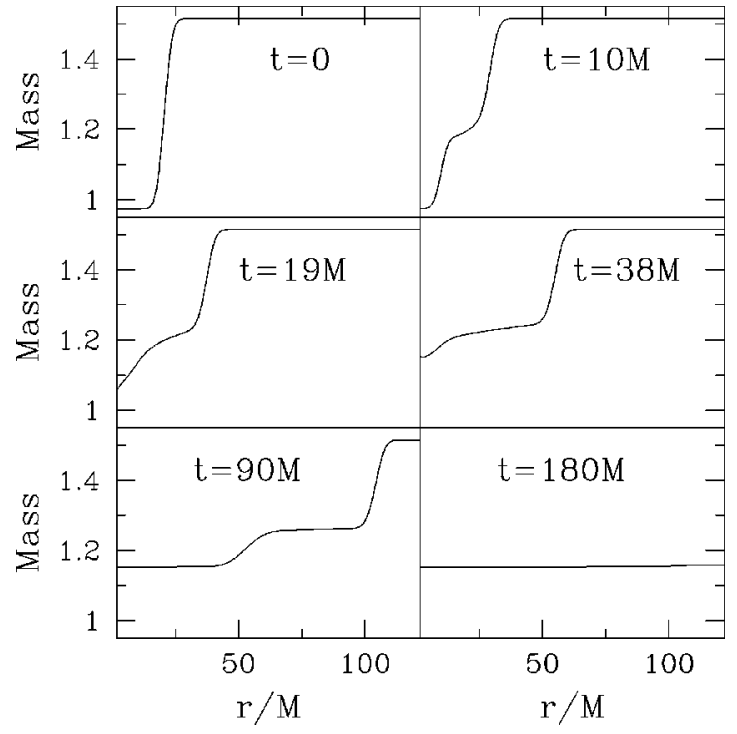

FIG. 13. Misner-Sharp mass, measured in units of $M$, as a function of radius at selected times for an evolution of the EinsteinKlein-Gordon system. Here $M$ is the mass of the background solution. See text for details.

outgoing quantities of the neighboring domain.

In Fig. 13 we plot the mass contained within radius $r$ as a function of $r$ for selected times. Initially the mass of the black hole is $0.97 \mathrm{M}$ and the mass of the entire spacetime is $1.52 M$. The scalar field energy concentrated near $r=20 M$ accounts for $0.55 \mathrm{M}$. As the evolution proceeds, the initial Gaussian scalar field pulse divides into incoming and outgoing pieces. The outgoing piece propagates to infinity, while the incoming piece is partially reflected off the Schwarzschild potential and partially swallowed by the black hole. At $t=90 M$ the mass of the black hole has reached its final value of $1.15 \mathrm{M}$, and the initial outgoing pulse and the reflected pulse have not yet reached the outer boundary of the domain. By $t=180 M$ the remaining scalar radiation has left the domain.

Figure 14 shows the coordinate radius and the areal radius of the apparent horizon versus time. The area of the horizon increases between $t=15 \mathrm{M}$ and $t=30 \mathrm{M}$ as the scalar field pulse falls into the black hole, and the areal radius asymptotically approaches the value $2.31 \mathrm{M}$, which is twice the mass of the final black hole as expected. Unlike the areal radius, the coordinate radius decreases with time until $t$ $=10 \mathrm{M}$, after which it increases and eventually asymptotes to $r=2.66 \mathrm{M}$. One can see from the figure that on the initial slice the coordinate $r$ is nearly areal, as it would be for the Kerr-Schild background solution without a scalar field. At late times the deviation from an areal radial coordinate is large.

Figure 15 shows the norm of the Hamiltonian constraint as a function of time for the same evolution, as well as for other evolutions with different spatial resolutions but the same $\Delta t$. The plots exhibit exponential convergence, even with nontrivial dynamics and multiple domains. There is, however, a small failure of convergence in the highest resolutions around $t=120 \mathrm{M}$. This is because the boundary con- 


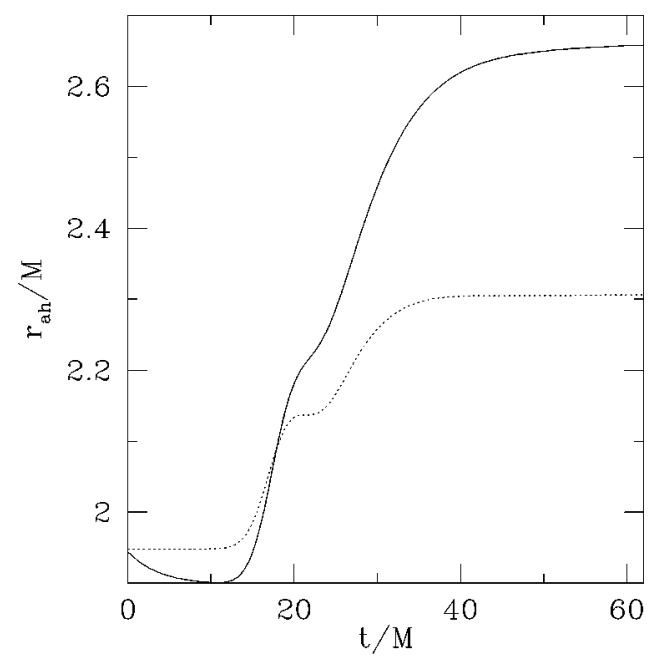

FIG. 14. Coordinate radius (solid line) and areal radius (dotted line) of the apparent horizon as a function of time for the evolution shown in Fig. 13.

dition (2.25), which is applied to $U_{r}^{-}$and $U_{\phi}^{-}$at the outer boundary $r_{b}$, is not strictly correct while a wave is passing through the boundary, and the error this introduces scales like $r_{b}^{-2}$. We have verified this by repeating the evolutions from Fig. 15 with the inner boundary a factor of two closer, at $60 M$. This is shown in Fig. 16. The resolution per subdomain is the same as in Fig. 15, but we use 4 equal-sized subdomains instead of 8 . The small nonconvergent feature in Fig. 16 is approximately a factor of four larger than in Fig. 15 , and occurs at an earlier time, $t=60 \mathrm{M}$, because the wave pulse reaches the outer boundary a factor of two earlier.

\section{DISCUSSION}

We have found that, at least for spherical symmetry, applying PSC methods to hyperbolic formulations of general relativity can achieve stable evolutions of black holes with

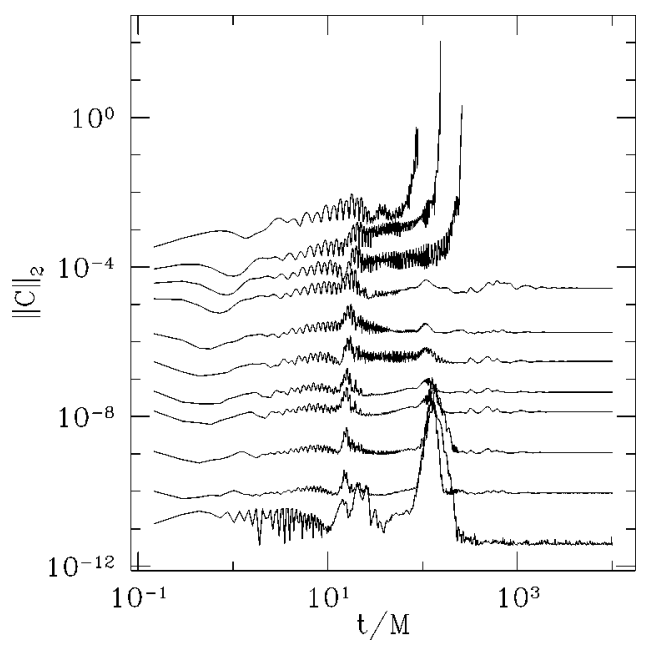

FIG. 15. Norm of the Hamiltonian constraint (2.20a) as a function of time for the evolution shown in Fig. 13 and for coarser evolutions with $12,16,18,20,24,27,30,32,36$, and 40 spectral coefficients per domain.

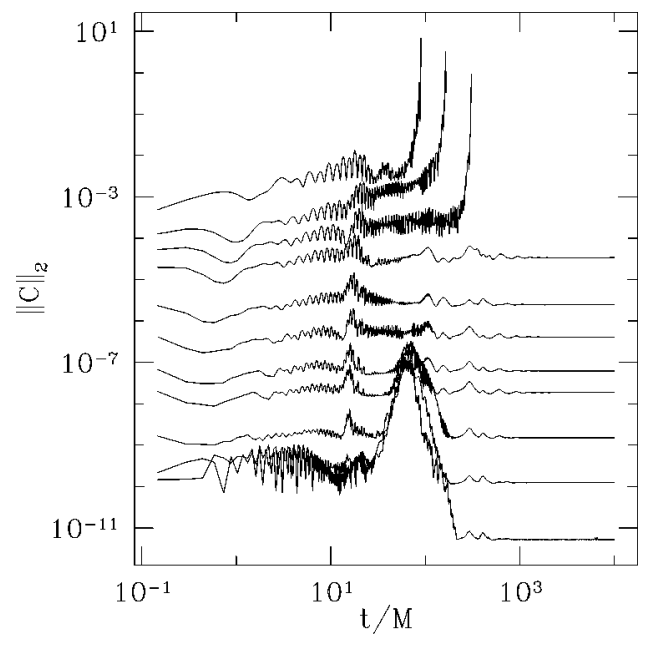

FIG. 16. Same as Fig. 15 except each evolution has an outer boundary radius of $60 \mathrm{M}$ instead of $120 \mathrm{M}$.

horizon excision. Excision itself is trivial as long as one uses a formulation in which all characteristic speeds are causal. Using realistic elliptic gauge conditions, our evolutions are limited only by linearly growing gauge modes that converge exponentially to zero with increasing resolution. These modes create no difficulty for long-term simulations because a small increase in resolution enables one to run much farther in time. We note that even when errors grow exponentially in time (e.g., Fig. 4), the high accuracy provided by PSC allows us to evolve to times of hundreds or sometimes thousands of $M$.

A hyperbolic formulation of Einstein's equations provides a straightforward way in which to formulate and implement boundary conditions using the complete set of characteristic eigenfields provided by hyperbolicity. In principle, our method can be applied to any hyperbolic formulation, but so far we have only used the EC system. We have not investigated whether PSC can be used with non-hyperbolic formulations of Einstein's equations such as the ADM formulation, as this would require a different treatment of the boundary conditions. It is entirely possible, however, that one might find boundary conditions that result in stable evolutions for such a formulation.

There has also been some concern about using hyperbolic representations of general relativity with complicated gauge conditions. This is because hyperbolic formulations of Einstein's equations formally require the gauge quantities (shift and densitized lapse in the case of EC) to be prescribed functions of space and time, and not evolved quantities that couple to the fundamental variables. However, as long as the gauge variables are held fixed during each entire time step, as discussed by [53] and [54], we find no fundamental difficulty in applying elliptic gauge conditions during our simulations.

By using the constraints as boundary conditions on the hyperbolic evolution equations, we have found that one can improve evolutions of the EC system. We have found similar improvement for finite-difference evolutions as well. Even in the general 3D case, applying constraint boundary conditions on the metric variables is straightforward. However, casting 


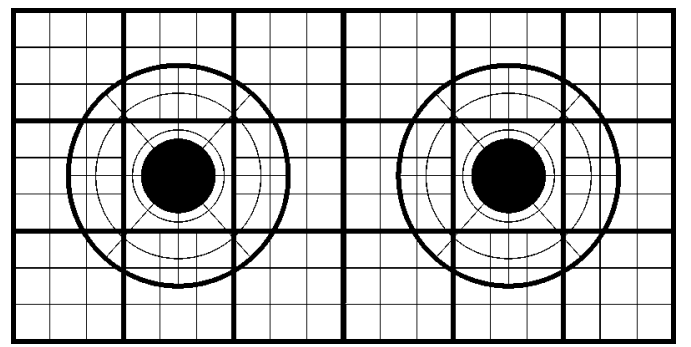

FIG. 17. Two-dimensional illustration of multiple computational domains that might be used to solve the binary black hole problem. Each hole is surrounded by a single domain in the shape of a spherical shell. Multiple cubical domains overlap the spherical shells. There are 16 shown here in 2 dimensions, with the cubes containing the black holes excised.

the Hamiltonian and momentum constraints as boundary conditions may be more difficult, because unlike in spherical symmetry, these constraints involve contractions of derivatives of fundamental variables.

The numerical techniques discussed in this paper should be generalizable to three spatial dimensions. For PSC evolutions of two black holes with excised horizons it will be necessary to use multiple computational domains (see Fig. 17). In spherical symmetry, we have shown how multiple domains can be easily implemented in a natural way by using characteristic fields to provide inter-domain boundary conditions. This method is directly applicable to abutting domains in $3 \mathrm{D}$, and the extension from abutting domains to overlapping domains is straightforward [55]. Work on 3D black hole evolutions using PSC is in progress.

\section{ACKNOWLEDGMENTS}

This work was supported in part by NSF grants PHY9800737 and PHY-9900672 and NASA grant NAG5-7264 to Cornell University, and NSF grants PHY-9802571 and PHY9988581 to Wake Forest University. Computations were performed on the National Computational Science Alliance SGI Origin2000, and on the Wake Forest University Department of Physics IBM SP2 with support from an IBM SUR grant.

\section{APPENDIX: COORDINATE SYSTEMS}

It is convenient to choose a coordinate system in which the Schwarzschild geometry is time-independent. Furthermore, since we wish to include the apparent horizon in our computational domain, we must choose coordinates such that the spacelike slices labeled by constant values of coordinate $t$ penetrate the horizon and are nonsingular there. Here we list several coordinate systems that satisfy these properties.

\section{Kerr-Schild coordinates}

In this coordinate system, also referred to as ingoing Eddington-Finkelstein coordinates [45], ingoing null rays have unit coordinate speed. In addition, the radial coordinate $r$ is chosen such that $4 \pi r^{2}$ is the surface area of a sphere at that radius. In this coordinate system the Schwarzschild solution takes the form

$$
\begin{aligned}
g_{r r} & =1+\frac{2 M}{r}, \\
g_{T} & =1, \\
\tilde{\alpha} & =\left(1+\frac{2 M}{r}\right)^{-1}, \\
\beta^{r} & =\frac{2 M}{r}\left(1+\frac{2 M}{r}\right)^{-1}, \\
K_{r r} & =-\frac{2 M}{r^{2}}\left(1+\frac{M}{r}\right)\left(1+\frac{2 M}{r}\right)^{-1 / 2}, \\
K_{T} & =\frac{2 M}{r^{2}}\left(1+\frac{2 M}{r}\right)^{-1 / 2}, \\
f_{r r r} & =\frac{1}{r}\left(4+\frac{7 M}{r}\right), \\
f_{r T} & =\frac{1}{r},
\end{aligned}
$$

where $M$ is the mass of the hole. The event horizon is coincident with the apparent horizon and is located at $r=2 \mathrm{M}$.

\section{Painlevé-Gullstrand coordinates}

In this coordinate system [26,56-58] the spatial threemetric is flat and the Schwarzschild solution is particularly simple:

$$
\begin{aligned}
g_{r r} & =1, \\
g_{T} & =1, \\
\tilde{\alpha} & =1, \\
\beta^{r} & =\sqrt{\frac{2 M}{r}}, \\
K_{r r} & =-\sqrt{\frac{M}{2 r^{3}}}, \\
K_{T} & =\sqrt{\frac{2 M}{r^{3}},} \\
f_{r r r} & =\frac{4}{r}, \\
f_{r T} & =\frac{1}{r} .
\end{aligned}
$$

The horizon is again located at $r=2 M$. 
3. Harmonic time slicing, areal radial coordinates

If one requires the time coordinate to satisfy $\square t=0$, the radial coordinate $r$ to correspond to the areal radius, and the coordinate system to be regular at the horizon, then the Schwarzschild solution takes the form $[59,60]$

$$
\begin{aligned}
g_{r r} & =\left(1+\frac{2 M}{r}\right)\left(1+\frac{4 M^{2}}{r^{2}}\right), \\
g_{T} & =1, \\
\tilde{\alpha} & =\left(1+\frac{2 M}{r}\right)^{-1}\left(1+\frac{4 M^{2}}{r^{2}}\right), \\
\beta^{r} & =\frac{4 \tilde{\alpha} M^{2}}{r^{2}}, \\
K_{r r} & =-\frac{4 M^{2}}{r^{3}} \sqrt{\tilde{\alpha}}\left(2+\frac{3 M}{r}+\frac{4 M^{2}}{r^{2}}+\frac{4 M^{3}}{r^{3}}\right), \\
K_{T} & =\frac{4 M^{2}}{r^{3}} \sqrt{\tilde{\alpha}}, \\
f_{r T} & =\frac{1}{r} \cdot \\
f_{r r r} & =\frac{4}{r}+\frac{7 M}{r^{2}}+\frac{12 M^{2}}{r^{3}}+\frac{20 M^{3}}{r^{4}},
\end{aligned}
$$

The horizon is at $r=2 M$.

\section{Fully harmonic coordinates}

The Schwarzschild solution can also be written in a coordinate system where all coordinates satisfy $\square x^{\mu}=0$ and are regular at the event horizon $[59,60]$ :

$$
\begin{aligned}
g_{r r} & =1+\epsilon+\epsilon^{2}+\epsilon^{3}, \\
g_{T} & =\left(1+\frac{M}{r}\right)^{2}, \\
\tilde{\alpha} & =\left(1+\frac{M}{r}\right)^{-1}\left(1+\epsilon^{2}\right)^{-1}\left(1+\frac{3 M}{r}\right)^{-1},
\end{aligned}
$$

$$
\beta^{r}=\epsilon^{2}\left(1+\frac{M}{r}\right)\left(1+\epsilon^{2}\right)^{-1}\left(1+\frac{3 M}{r}\right)^{-1}
$$$$
K_{r r}=-\frac{K_{T}}{g_{T}}\left(2+\frac{3 \epsilon}{2}+\epsilon^{2}+\frac{\epsilon^{3}}{2}\right),
$$$$
K_{T}=\frac{4 M^{2}}{r^{3}} \sqrt{\tilde{\alpha}}
$$$$
f_{r r r}=\frac{1}{g_{T}}\left[\frac{4}{r}\left(1+\epsilon^{2}\right)+\frac{M}{r^{2}}\left(11-2 \epsilon+9 \epsilon^{2}\right)\right],
$$

$$
f_{r T}=\frac{1}{r}+\frac{M}{r^{2}}
$$

where

$$
\epsilon \equiv \frac{2 M}{r}\left(1+\frac{M}{r}\right)^{-1}
$$

Here the horizon is located at $r=M$.
[1] M. W. Choptuik, Phys. Rev. Lett. 70, 9 (1993).

[2] S. L. Shapiro, S. A. Teukolsky, and J. Winicour, Phys. Rev. D 52, 6982 (1995).

[3] S. F. Portegies Zwart and S. L. W. McMillan, Astrophys. J. Lett. 528, L17 (2000).

[4] E. E. Flanagan and S. A. Hughes, Phys. Rev. D 57, 4566 (1998).

[5] W. Landry and S. A. Teukolsky, gr-qc/9912004.

[6] M. Shibata and K. Uryu, Phys. Rev. D 61, 064001 (2000).

[7] R. Arnowitt, S. Deser, and C. W. Misner, in Gravitation: An Introduction to Current Research, edited by L. Witten (Wiley, New York, 1962), pp. 227-265.

[8] S. Frittelli and O. Reula, Commun. Math. Phys. 166, 221 (1994)

[9] Y. Choquet-Bruhat and J. W. York, Jr., C. R. Seances Acad. Sci., Ser. 1 A321, 1089 (1995).
[10] A. Abrahams, A. Anderson, Y. Choquet-Bruhat, and J. W. York, Jr., Phys. Rev. Lett. 75, 3377 (1995).

[11] C. Bona, J. Massó, E. Seidel, and J. Stela, Phys. Rev. Lett. 75, 600 (1995).

[12] M. H. P. M. van Putten and D. M. Eardley, Phys. Rev. D 53, 3056 (1996).

[13] S. Frittelli and O. A. Reula, Phys. Rev. Lett. 76, 4667 (1996).

[14] H. Friedrich, Class. Quantum Grav. 13, 1451 (1996).

[15] F. B. Estabrook, R. S. Robinson, and H. D. Wahlquist, Class. Quantum Grav. 14, 1237 (1997).

[16] A. Anderson, Y. Choquet-Bruhat, and J. W. York, Jr., Topol. Meth. Nonlin. Anal. 10, 353 (1997).

[17] M. Alcubierre, B. Brugmann, M. Miller, and W.-M. Suen, Phys. Rev. D 60, 064017 (1999).

[18] S. Frittelli and O. A. Reula, J. Math. Phys. 40, 5143 (1999).

[19] A. Anderson and J. W. York, Jr., Phys. Rev. Lett. 82, 4384 (1999). 
[20] T. W. Baumgarte and S. L. Shapiro, Phys. Rev. D 59, 024007 (1999).

[21] M. A. Scheel et al., Phys. Rev. D 58, 044020 (1998).

[22] E. Seidel and W.-M. Suen, Phys. Rev. Lett. 69, 1845 (1992).

[23] M. Alcubierre and B. F. Schutz, J. Comput. Phys. 112, 44 (1994).

[24] M. A. Scheel et al., Phys. Rev. D 56, 6320 (1997).

[25] G. B. Cook et al., Phys. Rev. Lett. 80, 2512 (1998).

[26] C. Gundlach and P. Walker, Class. Quantum Grav. 16, 991 (1999).

[27] L. Lehner, M. Huq, and D. Garrison, gr-qc/0004065.

[28] A. M. Abrahams et al., Phys. Rev. Lett. 80, 1812 (1998).

[29] N. T. Bishop, R. Gómez, L. Lehner, and J. Winicour, Phys. Rev. D 54, 6153 (1996).

[30] R. Gomez et al., Phys. Rev. Lett. 80, 3915 (1998).

[31] N. T. Bishop et al., in Black Holes, Gravitational Radiation and the Universe, edited by B. R. Iyer and B. Bhawal (Kluwer, Dordrecht, 1998), Chap. 24.

[32] M. Alcubierre, Phys. Rev. D 55, 5981 (1997)

[33] S. A. Orszag, J. Atmos. Sci. 28, 1074 (1971).

[34] J. P. Boyd, Chebyshev and Fourier Spectral Methods (Springer-Verlag, Berlin, 1989).

[35] C. Canuto, M. Y. Hussaini, A. Quarteroni, and T. A. Zang, Spectral Methods in Fluid Dynamics (Springer-Verlag, Berlin, 1988).

[36] B. Fornberg, A Practical Guide to Pseudospectral Methods (Cambridge University Press, New York, 1996).

[37] S. Bonazzola, E. Gourgoulhon, and J.-A. Marck, J. Comput. Appl. Math. 109, 892 (1999).

[38] J. Novak and J. M. Ibáñez, Astrophys. J. 533, 392 (2000).

[39] S. Bonazzola, E. Gourgoulhon, and J.-A. Marck, Phys. Rev. Lett. 82, 892 (1999).

[40] L. E. Kidder and L. S. Finn, Phys. Rev. D (to be published), gr-qc/9911014.
[41] J. Frauendiener, J. Comput. Appl. Math. 109, 475 (1999).

[42] C. Gundlach, Phys. Rev. D 57, 863 (1998).

[43] S. Bonazzola et al., in ICOSAHOM'95, Proceedings of the Third International Conference on Spectral and High Order Methods, edited by A. V. Ilin and L. R. Scott (Houston Journal of Mathematics, Houston, 1996), pp. 3-19.

[44] R. A. Bartnik and A. H. Norton, SIAM J. Sci. Comput. (to be published), gr-qc/9904045.

[45] R. L. Marsa and M. W. Choptuik, Phys. Rev. D 54, 4929 (1996).

[46] P. Anninos et al., Phys. Rev. D 51, 5562 (1995).

[47] L. L. Smarr and J. W. York, Jr., Phys. Rev. D 17, 2529 (1978).

[48] D. Garfinkle, C. Gundlach, J. Isenberg, and N. O. Murchadha, gr-qc/0003113.

[49] D. Garfinkle and C. Gundlach, Class. Quantum Grav. 16, 4111 (1999).

[50] C. W. Misner, K. S. Thorne, and J. A. Wheeler, Gravitation (Freeman, New York, New York, 1973).

[51] J. W. York, Jr., in Sources of Gravitational Radiation, edited by L. L. Smarr (Cambridge University Press, Cambridge, England, 1979), pp. 83-126.

[52] S. B. Baden, D. Shalit, and R. B. Frost, "KeLP User Guide," http://www.cse.ucsd.edu/groups/hpcl/scg/kelp.

[53] J. W. York, Jr. (private communication).

[54] C. Bona, J. Massó, E. Seidel, and J. Stela, Phys. Rev. D 56, 3405 (1997).

[55] D. A. Kopriva, SIAM (Soc. Ind. Appl. Math.) J. Sci. Stat. Comput. 10, 120 (1989).

[56] P. Painlevé, Acad. Sci., Paris, C. R. 173, 677 (1921).

[57] A. Gullstrand, Ark. Mat., Astron. Fys. 16, 1 (1922).

[58] K. Martel and E. Poisson, gr-qc/0001069.

[59] C. Bona and J. Massó, Phys. Rev. D 38, 2419 (1988).

[60] G. B. Cook and M. A. Scheel, Phys. Rev. D 56, 4775 (1997). 